\title{
基于有机小分子的三磷酸腺苷苂光传感器研究进展
}

\author{
张继东*, $a$ 张 俊 ${ }^{b}$ 严 瞻 ${ }^{b}$ 谢娟平 ${ }^{c}$ \\ ( ${ }^{a}$ 安康学院化学化工学院 陕西省富硒食品质量监督检验中心 陕西安康 725000) \\ ( ${ }^{b}$ 西北大学化学与材料科学学院 合成与天然功能分子化学教育部重点实验室 西安 710127) \\ ( 安康学院医学院 陕西安康 725000)
}

\begin{abstract}
摘要 三磷酸腺苷(ATP)是各种活细胞内普遍存在的一种高能磷酸化合物, 在能量的储存, 细胞呼吸作用和酶催化反 应等生物活动过程中扮演着重要作用. 因此, 对于 ATP 在生物体内的研究至关重要. 苂光检测技术具有操作方便、选 择性好和灵敏度高等优点, 设计合成高效的 ATP 苂光传感器是近年来生物化学和分析化学领域的研究热点. 根据 ATP 荧光传感器的结构特点和识别原理, 将 ATP 荧光传感器分为金属 Zn(II)作为键合位点型识别, 其它金属离子作为键合 位点型识别和静电或氢键作用型识别. 基于有机小分子荧光传感器, 综述了近年来国内外 ATP 荧光传感器在分子设计 与应用方面的研究进展, 并展望了其发展趋势.
\end{abstract}

关键词＼cjkstart苂光传感器; 三磷酸腺苷; 生物成像; 有机小分子

\section{Recent Progress in Fluorescent Probes for Adenosine Triphosphate Based on Small Organic Molecules}

\author{
Zhang, Jidong ${ }^{*, a} \quad$ Zhang, Jun $^{b} \quad$ Yan, Zhan $^{b} \quad$ Xie, Juanping ${ }^{c}$ \\ ( ${ }^{a}$ Quality Supervision and Inspection Centre of Se-enriched Food of Shaanxi Province, School of Chemistry \& Chemical \\ Engineering, Ankang Univerisity, Ankang, Shaanxi 725000) \\ $\left({ }^{b}\right.$ Key Laboratory of Synthetic and Natural Functional Molecule Chemistry of the Ministry of Education, College of \\ Chemistry \& Materials Science, Northwest University, Xi'an 710127) \\ ( ${ }^{c}$ School of Medicine, Ankang Univerisity, Ankang, Shaanxi 725000)
}

\begin{abstract}
Adenosine triphosphate (ATP) is a high-energy phosphate compound commonly existing in various living cells, which plays important roles in the biological activities such as energy storage, cell respiration and enzyme catalytic reactions. Therefore, it is very crucial to ATP research in bio-organism. Fluorescence detection techniques has the advantages of convenient operation, good selectivity and high sensitivity, etc. In recent years, the design and synthesis of efficient ATP fluorescence sensors have become a research focus in the fields of biochemistry and analytical chemistry. According to the structure characteristics, ATP fluorescence chemosensors are categorized as Zn(II) metal ions as recognition of bonding site type, other metal ions as recognition of bonding site type and electrostatic or hydrogen bond as interaction type. Based on organic small molecule fluorescence sensors, the recent progress in research of ATP fluorescence sensors in molecular design and application is reviewed, and the prospects for their development are discussed.
\end{abstract}

Keywords fluorescent chemosensor; adenosine triphosphate; biological imaging; small organic molecules

核苷磷酸衍生物是广泛存在于活细胞中的物质, 在 各种生命体中发挥着非常重要的作用. 三磷酸腺苷 (ATP)作为细胞内的能量货币，在有机体各种新陈代谢
循环中普遍存在 ${ }^{[1,2]}$. 体内和体外 ATP 含量的确定能够 为理解精准的生物过程提供有价值的信息. ATP 作为信 号分子，调节细胞运动、神经传递和离子运输. 另外，

\footnotetext{
* Corresponding author. E-mail: akuzjd@aku.edu.cn

Received May 12, 2019; revised June 27, 2019; published online July 17, 2019.

Project supported by the Youth Foundation of Shaanxi Provincial Science \& Technology Department (No. 2019JQ-504), the Major Scientific Research Projects of the Leading Industry of Ankang City (No. 2016AKZDCY002), the Doctor's Initial Funding of Ankang University (No. 2018AYQDZR06), the Key Laboratory of Se-enriched Products Development and Quality Control, Ministry of Agriculture (No. Se-2018B02), and the Shaanxi Provincial Innovation Experiment Program for University Students (No. 201839032).

陕西省科技厅青年基金(No. 2019JQ-504)、安康市主导产业重大科研攻关(No. 2016AKZDCY002)、安康学院博士启动基金(No. 2018AYQDZR06)、农 业部富硒产品开发国家地方联合工程实验室开放课题(No. Se-2018B02)、陕西省大学生创新创业训练计划(No. 201839032)资助项目.
} 
ATP 也参与生物体内许多酶反应过程, 如激酶催化的蛋 白磷酸化过程、糖基转移酶催化过程等 ${ }^{[3,4]}$. ATP 在体内 的含量也与各种疾病密切相关, 包括心血管疾病、帕金 森氏症和低血糖等疾病. 鉴于 ATP 在生命体内的上述重 要意义, 准确定性和定量检测 ATP 技术是非常需要的 ${ }^{[5]}$.

近年来, 苂光技术被分析化学和生物化学科学家所 青睐 ${ }^{[6 ~ 8]}$. 苂光传感器是建立在光谱化学测量技术基础 上的一种将分析对象的化学信息以荧光信号的方式表 达出来的一种检测技术 ${ }^{[4,9]}$. 利用荧光传感技术对有机 磷酸类阴离子, 如 ATP, 二磷酸腺苷(ADP), 磷酸腺苷 (AMP)和三磷酸鸟苷(GTP) 等物质的检测在生物体内是 非常重要的研究方向. 在各种 ATP 的检测方法中, 如高 效液相色谱法, 离子色谱法和质谱法等, 常需要特定的 设备, 操作复杂, 检测准确度较差. 苂光检测法因操作 简单、灵敏度高、选择性好以及可原位实时定量监测等 优点而受到科研工作者广泛关注 ${ }^{[10 ~ 13]}$.

此外, 苂光传感器可以用于检测细胞内生物分子的 波动水平, 其检测结果在一定程度上可以直接反映人体 健康状况 ${ }^{[14 ~ 16]}$. 因此, 用于体内和体外检测的各种类型 的 ATP 荧光传感器的设计成为热点. 尽管 ATP 在细胞活 动中具有重要意义, 但相比较于其它一些生物活性离子 (如 $\mathrm{Zn}^{2+}, \mathrm{Ca}^{2+}$ ), ATP 荧光传感器的研究较少, 其主要原 因为 ATP 分子较难引起荧光发射. 一般情况下, ATP 荧 光传感器的识别大多是基于 $\mathrm{NH}$ 键和金属阳离子的络合 作用及 $\pi-\pi$ 相互作用. 其中, 含有两个金属中心结合位 点的 Zn-DPA 络合物[DPA, $N, N^{\prime}$-二(2-吡啶甲基)胺]被广 泛作为 ATP 识别基团 ${ }^{[10,17]}$. 近几年 ATP 荧光传感器得到 了快速的发展 ${ }^{[17,18]}$, 本文综述了基于有机小分子的 ATP 荧光传感器研究进展, 期望对该领域的研究工作者有所 帮助.

\section{1 金属 Zn(II)作为键合位点的化学传感器}

近年来, $\mathrm{Zn}(\mathrm{II})$-二甲基吡啶胺(Zn-DPA)结构单元广 泛用于构筑含磷酸基团阴离子苂光传感器. 主要原因是 二甲基吡啶胺能够与金属锌形成稳定的配合物, 其对含 磷酸基团阴离子有强的结合能力. 到目前为止, 2,2-二 甲基吡啶胺 $-\mathrm{Zn}(\mathrm{II})$ 配合物已被普遍认为是含磷酸类物质 传感器的特定识别单元. 因此, 2,2-二甲基吡啶胺-Zn(II) 配合物被用于构筑 ATP 苂光传感器.

2004 年, Hamachi 等 ${ }^{[19]}$ 报道了两种包含两个 $\mathrm{Zn}(\mathrm{II})$ 离子配位中心的二甲基吡啶胺结构苂光传感器 1 和 2 . 在各种阴离子存在的水溶液环境中, 其对磷酸类物质表 现出高选择性. 相比较于单磷酸类化合物, 其对 ATP, $\mathrm{ADP}$ 等焦磷酸类衍生物表现出强的结合能力. 传感器 $\mathbf{1}$

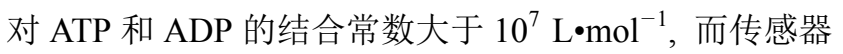

2 对 ATP 和 ADP 的结合常数分别是 $4.0 \times 10^{5}$ 和 $1.6 \times 10^{5}$ $\mathrm{L} \cdot \mathrm{mol}^{-1}$. 在磷酸类衍生物家族中, 包括在水溶液中磷 酸化的多肽物质, 传感器 2 不能区分特定的磷酸物质. 该研究结果为后来基于 DPA- $\mathrm{Zn}^{2+}$ 作为识别基团的 ATP 苂光传感器的发展打开了新的窗口.

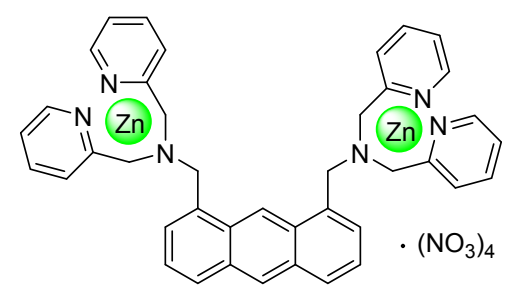

1

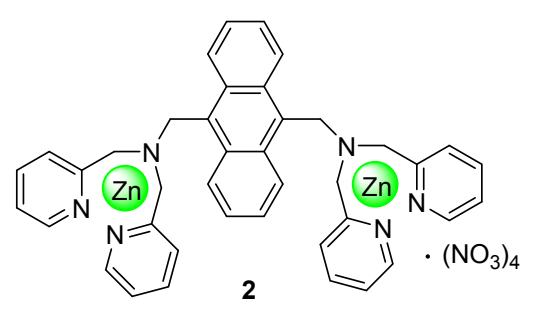

继该研究工作之后, 他们课题组又设计了一种新的 体系, 传感器 2 和 3 中嵌入一种糖基化胺的醋酸水凝胶 结构 ${ }^{[20]}$. 当加入 ATP 之后, 传感器 2 在 $435 \mathrm{~nm}$ 处产生 苂光发射，同时伴随着 2.6 倍的苂光强度的增加，其结 合常数大于 $10^{6} \mathrm{~L} \cdot \mathrm{mol}^{-1}$. 当传感器 3 在超分子水凝胶结 构(TSH 1)中被固定后, 加入 ATP、磷酸及其衍生物之后, 传感器 3 的苂光发射强度降低, 同时伴随着荧光发射的 红移. 不含磷酸结构的阴离子不能引起苂光发射强度的 改变. 值得注意的是超分子凝胶结构存在的疏水结构对 于客体分子诱导的荧光改变是重要的因素.
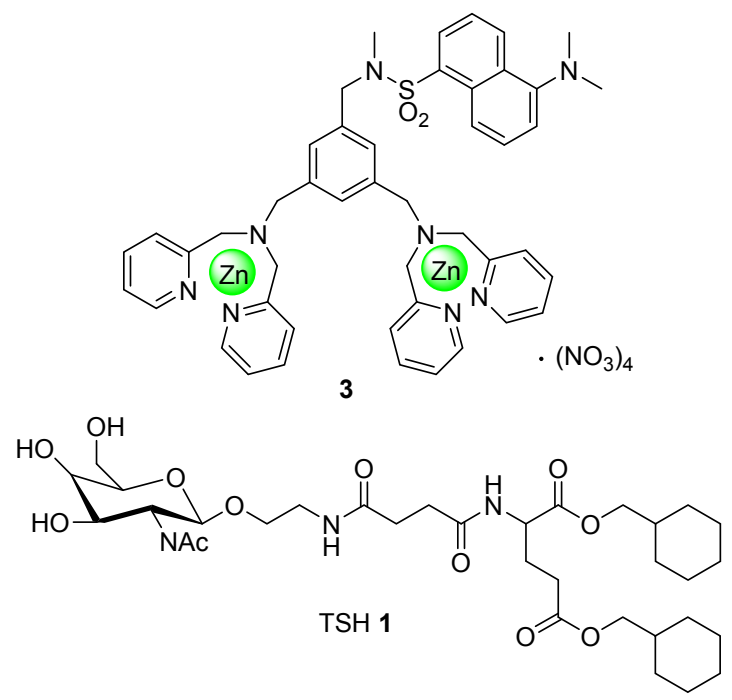

2006 年, Ojida 等 ${ }^{[21]}$ 开发了基于听啶结构的核苷焦 磷酸衍生物苂光传感器 $\mathbf{4}$ 和 $\mathbf{5}$, 可以在中性水溶液条件 下检测 ATP. 在 $\mathrm{pH}$ 为 7.2 时, 传感器 4 测定体系加入 
ATP 之后, 在 $468 \mathrm{~nm}$ 处的最大发射波长迅速降低, 并且 伴随着发射波长蓝移到 $441 \mathrm{~nm}$. 对于传感器 $\mathbf{5}$, 当加入 ATP 后, 荧光光谱被明显改变, 其苂光发射的颜色由最 初绿色的荧光变为蓝色. 传感器 4 和 5 对 ATP 的结合常 数分别为 $7.6 \times 10^{6}$ 和 $5.3 \times 10^{6} \mathrm{~L} \cdot \mathrm{mol}^{-1}$. 如 Scheme 1 所 示, 产生独特的发射波长位移归因于双核 $\mathrm{Zn}(\mathrm{II})$ 配合物 中第一个 $\mathrm{Zn}^{2+}$ 从叮啶氮原子上解离. 进一步研究发现, 该结构可以成功应用于一些生物体系中, 如荧光监测三 磷酸腺苷双磷酸酶的水解作用和糖基转移催化作用.

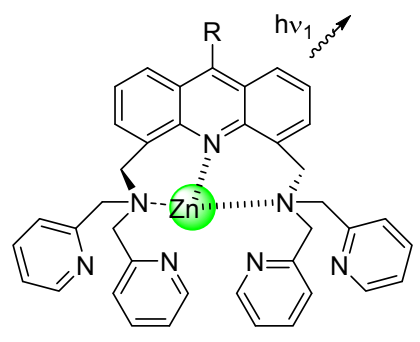

zn) $\sqrt{2 n}$

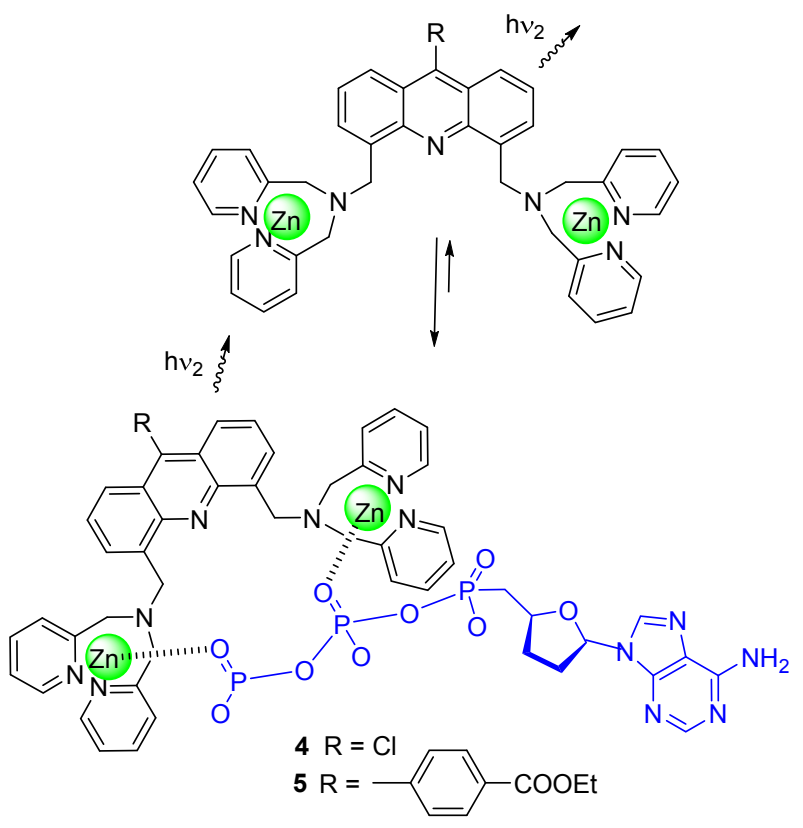

图式 1 传感器 4,5 与 ATP 的识别机理

Scheme 1 Structures of $\mathbf{4}$ and $\mathbf{5}$, and illustration of the dual-emission sensing mechanism with ATP

在同一年, 他们又报道了一种新的苂光传感器 $\mathbf{6}^{[22]}$, 其结构由 2,2 -二甲基吡啶胺-Zn(II)作为 ATP 检测的磷酸 基团结合位点和氧杂葱酮发光基团组成. 在中性水溶液 环境中, 随着 ATP 浓度的增加, 其激发波长在 322, 360 和 $407 \mathrm{~nm}$ 处随着浓度的增加发生改变, 同时出现两个 等吸收点. 传感器 6 对 ATP 表现出强的结合能力, 其结 合常数为 $4.5 \times 10^{5} \mathrm{~L} \cdot \mathrm{mol}^{-1}$. 对 ADP 的结合常数为

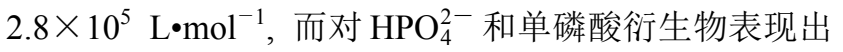

弱的结合能力.

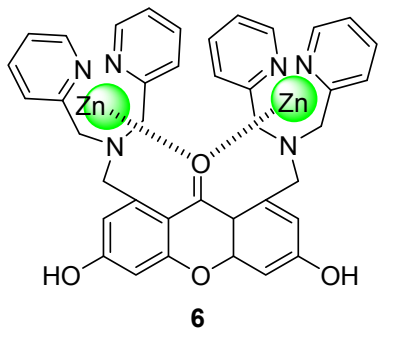

2007 年, Das 等 ${ }^{[23]}$ 报道了一种金属 Zn(II)配合物 7, 其在生理 $\mathrm{pH}$ 条件下的水溶液中能够选择性地结合 ATP. 传感器结构由对二甲胺基偶氮苯发色基团和二胺甲基 吡啶胺- $\mathrm{Zn}$ (II)识别基团组成. 在 4-(2-羟乙基)-1-哌嗪乙 磺酸(HEPES)缓冲溶液中, 传感器与 ATP 键合之后, 溶 液的颜色由淡黄色变为亮红色. 发射波长在 $463 \mathrm{~nm}$ 处, 传感器表现出弱的苂光发射, 当加入 ATP 之后, 其溶液 的荧光被部分淬灭. 而其它离子如 AMP, ADP, PPi, $\mathrm{PO}_{4}^{3-}, \mathrm{F}^{-}, \mathrm{Br}^{-}, \mathrm{Cl}^{-}, \mathrm{I}^{-}, \mathrm{CH}_{3} \mathrm{COO}^{-}, \mathrm{HSO}_{4}^{-}, \mathrm{SO}_{4}^{2-}$ 等没 有观察到溶液颜色的变化. 此外, 传感器 7 可以被用于 酵母细胞的标记试剂, 在自然光下能够进行显微成像检 测. 该传感器首次实现了水溶液中 ATP 的比色检测.

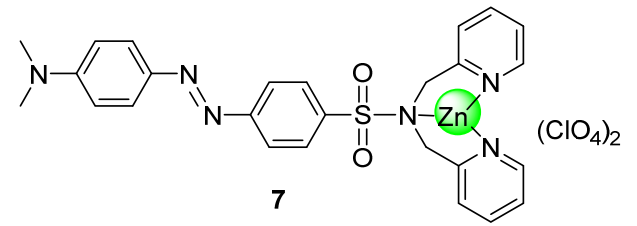

2011 年, 他们 ${ }^{[24]}$ 接着报道了一种 $\mathrm{Zn}$-环胺的环糊精 类轮烷结构 8, 其结构由 $\mathrm{Zn}(\mathrm{II})$ 配合物和 $\alpha$-环糊精组成. 在 $\mathrm{pH}=7.2$ 的 HEPES 缓冲溶液中, 当其它的竞争性阴离 子和生物中重要的核苷酸 $\mathrm{AMP}, \mathrm{ADP}, \mathrm{PPi}$ 和 $\mathrm{PO}_{4}^{3-}$ 等存 在时, 其与 ATP 能够优先选择性结合. 通过细胞实验研 究发现, 传感器 8 可以用于标记细胞内的 ATP, 同时可 以用于对革兰氏阳性菌和革兰氏阴性菌的着色. 更重要 的一点, 该荧光传感器对活的细胞没有表现出细胞毒 性, 可以用于细胞生长的动态研究. Gunning 等 ${ }^{[25]}$ 报道 了 $\mathrm{Zn}(\mathrm{II})$-大环多胺的三乙基苯结构的配合物 9, 其能够 在小分子磷酸类阴离子存在下, 在复杂的样品中能够选 择性地隔离 PPi 和 ATP, 实现磷肽类物质的检测.

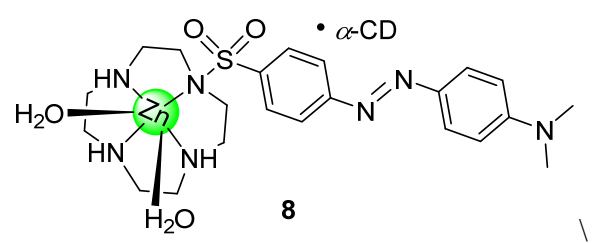

2010 年, Ojida 等 ${ }^{[17]}$ 报道了基于氧杂葱的比率性荧 光传感器 10 和 11, 在生命体系等复杂条件下, 可以精 确地测定生物体内 ATP. 其 turn-on 型荧光识别机理为键 


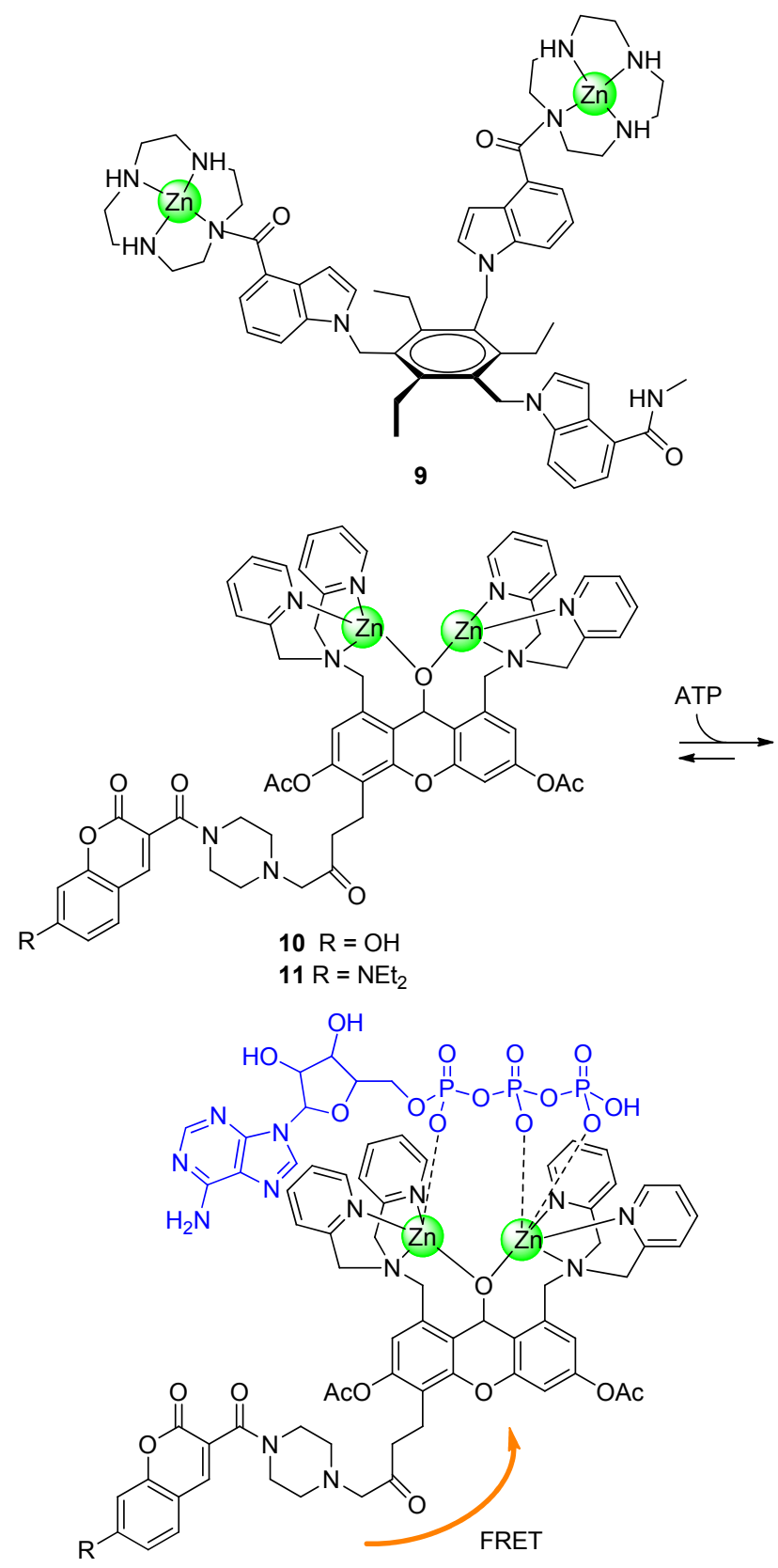

图式 2 传感器 10, 11 与 ATP 结合模式与识别机理

Scheme 2 Sensing mechanism and proposed binding mode between chemosensor 10, 11 and ATP

合诱导的苂光共振能量(FRET). 在传感器的设计过程 中, 引入香豆素荧光基团作为苂光共振能量转移的供 体, 双核 $\mathrm{Zn}(\mathrm{II})$ 的化合物作为苂光共振能量转移的受体. 在水溶液体系中, 其结合常数为 $K_{\text {app }} \approx 10^{6} \sim 10^{7} \mathrm{~L} \cdot$ $\mathrm{mol}^{-1}$. 单磷酸类物质、磷酸二脂和其它阴离子加入到传 感器所在的溶液中没有观察到荧光信号的改变. 该传感 器可以被用于实时地监测各种酶的反应, 如糖转移酶的 作用下糖的合成和蛋白激酶的作用下磷酸化作用. 进一 步荧光成像研究发现, 传感器 9 可以用于细胞内核苷磷 酸物质的苂光成像研究, 随 ATP 浓度的响应改变, 可以
揭示出细胞能量的水平. 该传感器的设计中，首次采用 结合诱导的 FRET 复原，该策略为设计基于 FRET 机理 的 ATP 苂光传感器提供有力依据.

2014 年, Butler 等 ${ }^{[26]}$ 报道了一种三足艮的 Zn(II)配 合物苂光传感器 12. 其能够在生理 $\mathrm{pH}$ 条件下实时的检 测三磷酸腺苷酶的活性. 传感器 $\mathbf{1 2}$ 对 ATP 的键合作用, 主要是依靠金属-配体的配位作用、氢键作用和 $\pi-\pi$ 堆 积作用。在其它竞争性阴离子存在的情况下，如 ADP, AMP, $\mathrm{HPO}_{4}^{2-}, \mathrm{PO}_{4}^{3-}, \mathrm{HCO}_{3}^{-}$依然能够检测 ATP. 没 有其它阴离子导致荧光信号改变的主要原因在于, 缺乏 与芳基脲官能团配位的结构大小一致性.在水溶液环境 中, 模拟细胞外流体环境, 传感器能够在 $\mathrm{mmol}$ 的水平 上比率性实时检测三磷酸催化酶水解的 ATP, 符合新陈 代谢 ATP 的浓度范围 $\left(1 \sim 5 \mathrm{mmol} \cdot \mathrm{L}^{-1}\right)$. 该传感器能够 实时监测磷酸酶催化的 ATP 水解作用, 遗憾的是该工作 没有在细胞水平上 ATP 检测的相关研究.

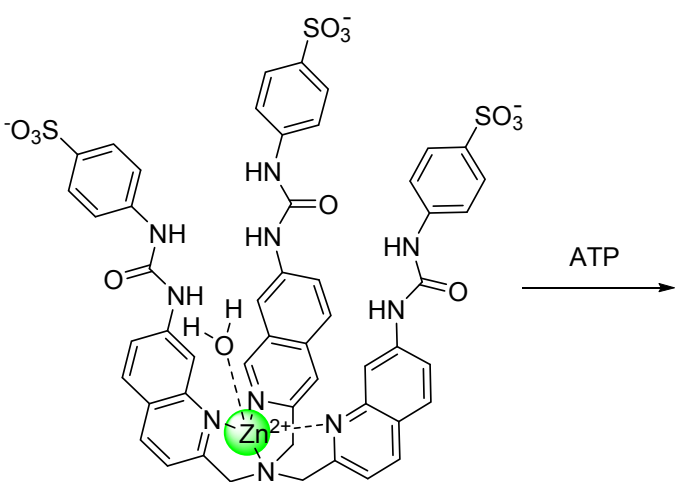

12

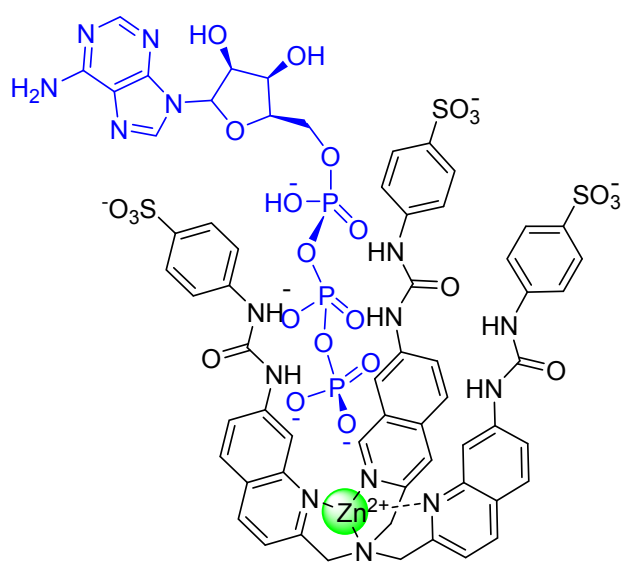

图式 3 传感器 12 与 ATP 的结合模式和识别机理 Scheme 3 Sensing mechanism and proposed binding mode between chemosensor $\mathbf{1 2}$ and ATP

2016 年, Anzenbacher 等 ${ }^{[27]}$ 报道了一种基于苯硼酸 连接 Zn- DPA 和领苯二酚类似物染料的 ATP 自组装荧 光传感器 13. 该传感器对寡聚磷酸类物质的选择性超 过单磷酸类物质，可用于可视化监测新陈代谢反应， ATP 水解生成焦磷酸和 AMP. 在邻苯二酚类似物染料 
的作用下, 传感器形成明亮的颜色. 研究选择传感器和 四种不同的染料, 邻苯二酚紫 $(P V)$ 、邻苯三酚红(PR)、 溴邻苯三酚红(BPR)和茜红素(ABS)在 HEPES 缓冲溶液 中进行结合能力的研究, 发现传感器对茜红素具有较强 的结合能力, 其结合常数为 $1.0 \times 10^{6} \mathrm{~L} \cdot \mathrm{mol}^{-1}$. 在各种 含磷酸根的阴离子存在的情况下, 如 ATP, AMP 和 PPi, 传感器表现出分析物特定的颜色变化, 能够定量监测磷 酸的浓度. 该研究工作是第一次使用超分子传感器在水 中定量确定磷酸类混合物. 这种策略为未来设计用于监 测各种阴离子混合物的传感器提供思路.

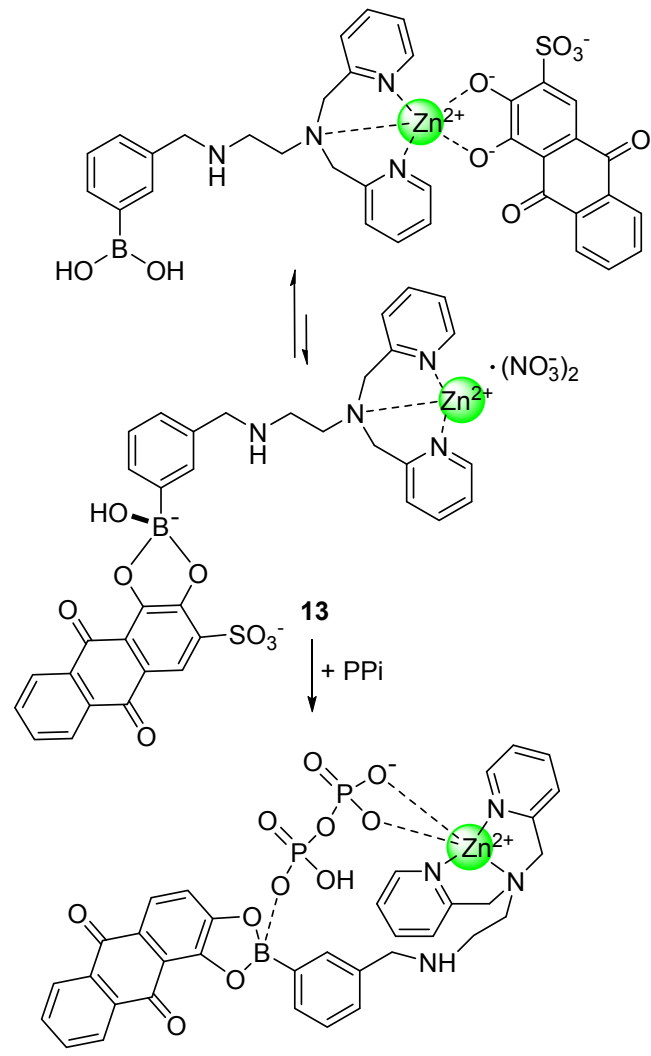

图式 4 传感器 13 与 PPi 的结合模式与识别机理 Scheme 4 Sensing mechanism and proposed binding mode between chemosensor $\mathbf{1 3}$ and PPi

2012 年, Ahn 等 ${ }^{[28]}$ 报道了一种基于 $\mathrm{Zn}(\mathrm{II})$-DPA (2,2二胺甲基吡啶)的双光子荧光传感器 14. 其能够在 $\mathrm{pH}$ 为 7.4 的生理状态下对 ATP 和 ADP 表现出高的选择性识别 能力. 当传感器所在溶液中存在 ATP 或 ADP 时, 溶液表 现出明显的荧光增强信号. 通过 ${ }^{1} \mathrm{H} N \mathrm{NR}$ 和荧光光谱研 究发现，苂光团和碱基之间的 $\pi-\pi$ 相互作用有助于对 ATP 的苂光选择性. 传感器 14 对 ATP 和 ADP 的结合常 数分别为 $6.2 \times 10^{6}$ 和 $3.2 \times 10^{5} \mathrm{~L} \cdot \mathrm{mol}^{-1}$, 对 ATP 的检测 浓度为 $1.0 \mu \mathrm{mol} \cdot \mathrm{L}^{-1}$. 通过细胞成像实验研究发现, 其 可以渗透细胞膜, 用于活的细胞中 ATP 和 ADP 的双光 子成像. 该研究工作是首次在活细胞中荧光检测 ATP 和 ADP 的传感器.

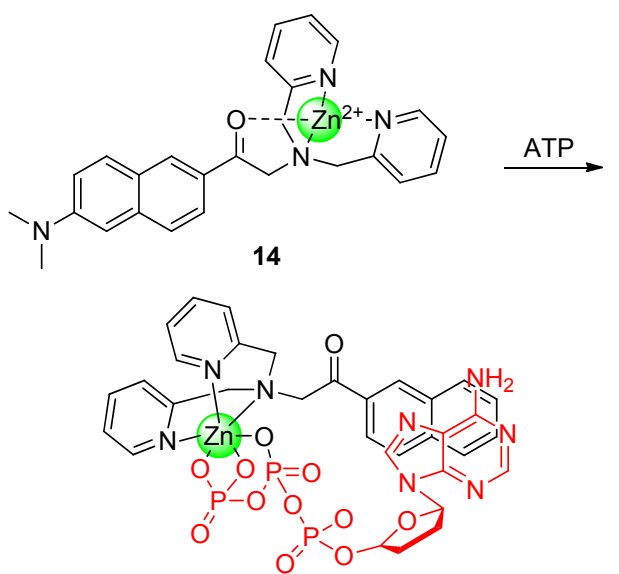

图式 5 传感器 14 与 ATP 的结合模式与识别机理 Scheme 5 Sensing mechanism and proposed binding mode between chemosensor 14 and ATP

2013 年, 鲁统部等 ${ }^{[29]}$ 设计合成了一种能够选择性 识别 ATP 和 ADP 的苂光传感器 15. 其结构由两个双核 $\mathrm{Zn}(\mathrm{II})$ 大环结构组成, 大环结构中含有两个对称的蒽环 和吡啶结构, 结构中的两个对称的蒽的片段能够与腺嘌 呤通过 $\pi-\pi$ 堆积相互作用形成一个三明治结构. 这种传 感器与 ATP 或 ADP 多重位点识别作用可以提高 ATP 或 ADP 的结合能力和选择性, 说明传感器对核苷磷酸化 合物选择性强弱可以通过增加传感器的识别位点调控. 涂涛等 ${ }^{[30]}$ 报道了运用简单的的三联吡啶锌的配合物通 过形成水凝胶识别 ATP 的苂光传感器 16. ATP 和三联吡 啶形成二组分的水凝胶的形成，通过苂光光谱研究和密 度泛函理论研究发现，其识别机理为以金属 $\mathrm{Zn}$ 为与磷 酸基金属配位相互作用，ATP 核酸的碱基与金属配合物 的芳环之间的 $\pi-\pi$ 堆积作用导致其自组装的形成. 该传 感器能够成功用于通过荧光共聚焦成像监测 HeLa 细胞 中的 ATP.

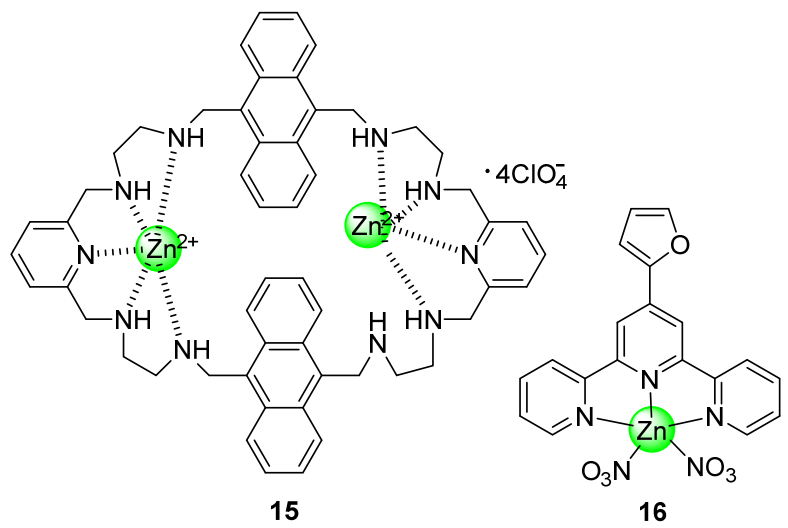

2010 年, Moro 等 ${ }^{[31]}$ 设计合成了一种由䒺二甲酰亚 胺和 $\mathrm{Zn}(\mathrm{II})-\mathrm{DPA}$ 组成的 ATP 荧光传感器 17. 其在 $\mu \mathrm{mol}$ 范围内在其它阴离子存在的体系中对 ATP 和 ADP 表现 出高的选择性. 作者推测其检测机理为, 在 $\mathrm{Zn}$ (II)存在 
的情况下，络合物表现出两种效应：首先，光致电子转 移(PET)效应的减弱, 导致传感器苂光减弱, 当 ATP 键 合到 Zn(II)-DPA 单元时导致 PET 效应被阻止, 苂光强度 增加. Yoon 等 ${ }^{[32]}$ 基于萠苂光基团和 $\mathrm{Zn}(\mathrm{II})-\mathrm{DPA}$ 识别基团 的 ATP 苂光传感器 $\mathbf{1 8}$, 其能够实现 ATP 和 ADP 的选择 性区分. 其识别原理为苂光团萠与腺嘌呤结构的自组 装, 导致苂光发射的增强. 邢国文等 ${ }^{[33]}$ 采用相似的识别 原理, 报道了结构相似的传感器 19.

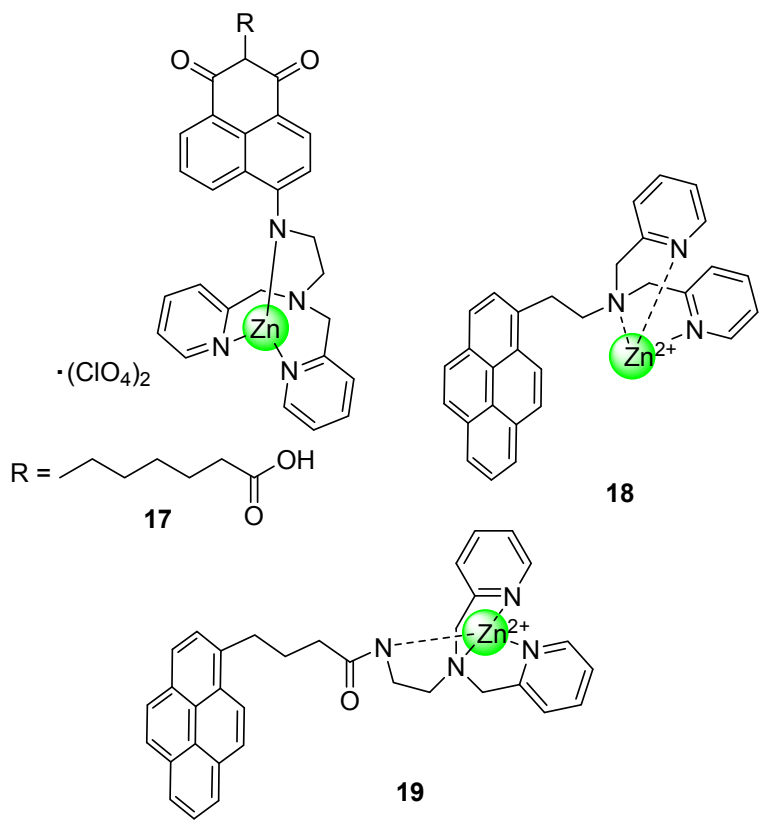

席夫碱结构具有对过渡金属离子很好的配位能力, 特别是对 $\mathrm{Cu}(\mathrm{II}) 、 \mathrm{Zn}(\mathrm{II})$ 等金属离子具有强的结合能力. 2012 年, Milione 等 ${ }^{[34]}$ 报道了一种结构简单的 $\mathrm{Zn}$ (II)配合 物作为 ATP 和 ADP 的苂光传感器 $\mathbf{2 0}$. 该传感器在水溶 液中能够对 ATP 和 ADP 选择性检测. 其主要依靠磷酸 基的电荷作用和腺苷与配体的氢键的作用. 在水溶液中 其苂光信号为 off-on 模式，通过 ${ }^{31} \mathrm{P} N M R,{ }^{1} \mathrm{H}$ NMR, 苂 光光谱和密度泛函理论(DFT)计算得到探针与 ADP/ATP 的结合模式, 其结果证明结构 20 可以成功应用于 ATP 和 ADP 选择性荧光检测.

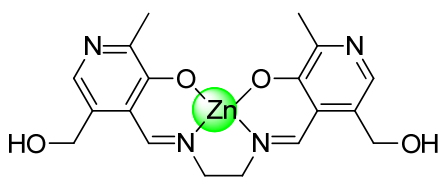

20

Sinha 等 ${ }^{[35]}$ 报道了基于香豆素硫醚席夫碱作为 turn-on 型 Zn(II)苂光传感器 21, 其能够用于 ATP 识别, 同时可以用于细胞内 ATP 的苂光成像研究. 通过 ${ }^{1} \mathrm{H}$ NMR, Job's 研究发现, 金属 Zn(II)与配体的结合方式为 $1: 1$ 的结合模式. 所形成的配合物对 ATP 的检测限为 $6.8 \mu \mathrm{mol} / \mathrm{L}$. Zelder 等 ${ }^{[36]}$ 报道了一种结构简单的遇 ATP
分解的 $\mathrm{Zn}(\mathrm{II})$ 苂光水杨醛席夫碱传感器 22, 23. 其在生 理条件下可以用于检测生物相关的二磷酸或三磷酸物 质. 通过试验研究发现, ATP 和 PPi 可以代替传感器结构 中的 $\mathrm{Zn}(\mathrm{II})$, 同时迅速水解释放出自由的席夫碱分子. 作者预测这种策略在未来其它样品检测的应用中是不 受限制的. 该研究工作采用分解模式检测 ATP, 该模式 为 ATP 的荧光检测提供新策略.

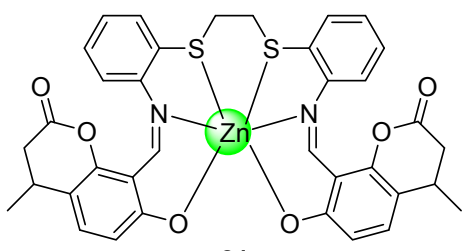

21

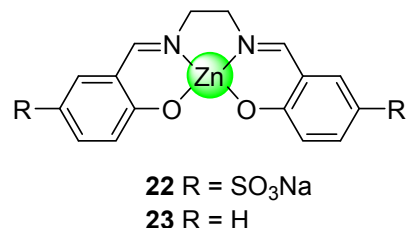

\section{2 其它金属离子作为键合位点的化学传感器}

尽管 Zn(II)的配合物对 ATP 具有强的结合能力, 根 据文献报道, $\mathrm{Cu}(\mathrm{II})$ 的配合物对磷酸盐表现出同样强度 的结合能力, $\mathrm{Cu}(\mathrm{II})$-磷酸作用为 ATP 的识别提供新的途 径. 2012 年, Kataev 等 ${ }^{[37]}$ 报道了两种苂光配体 24 和 25 , 在生理 $\mathrm{pH}$ 条件下, 能够与 $\mathrm{Cu}(\mathrm{II})$ 和含磷酸基团的衍生 物(ATP, ADP, AMP 和 PPi)形成三元复合物. 研究发现, 两个不同的配体与 $\mathrm{CuCl}_{2}$ 结合对 ATP, ADP 和 AMP 表现 出高的选择性, 实现比率性苂光识别, 而单独的一种配 合物不具有上述性质. 对每一种配体与 $\mathrm{CuCl}_{2}$ 制备的配 合物，其对磷酸类物质的苂光响应是不一样的，原因为 信号传导采用不同的机理：通过 $\pi-\pi$ 相互作用使得荧光 淬灭和分析物配位的分子内替代机理. 这是通过采用两 种苂光分子与金属离子配位，实现选择性识别的例子. 卢忠林等 ${ }^{[38]}$ 报道了一种 1,8 -菜二甲酰亚胺的 $\mathrm{Cu}(\mathrm{II})$ 配合 物 26, 其对 ATP 表现出强的选择性. 可以实现水溶液和 Hela 细胞中 ATP 的实时苂光成像检测.

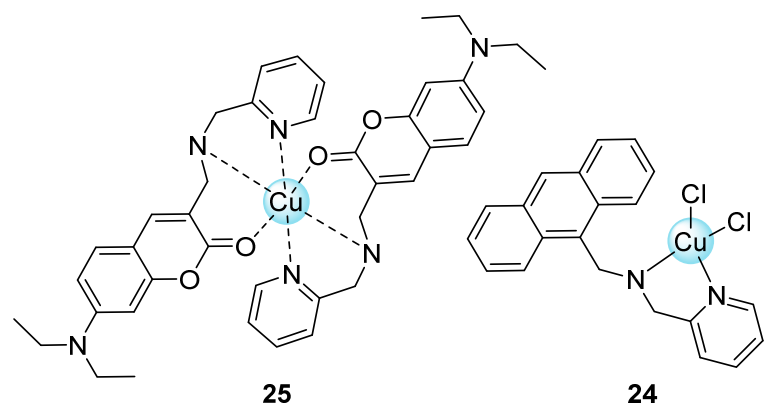




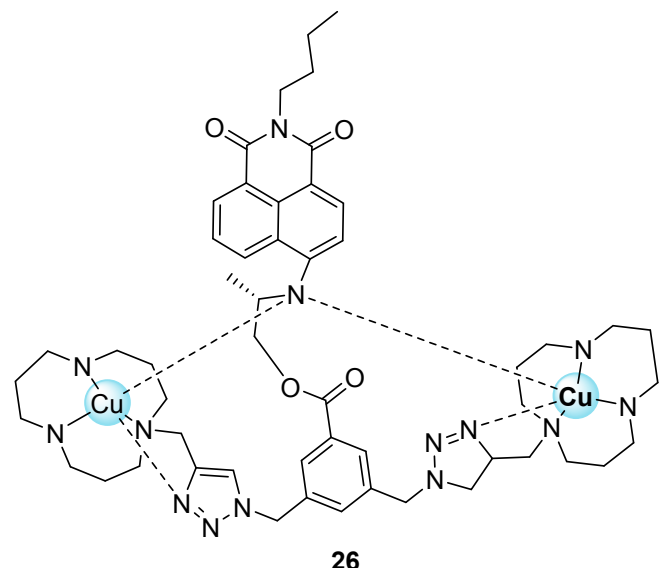

研究发现, $\mathrm{Ga}(\mathrm{III})$ 很容易配位到苯酚羟基部分的的 氧原子. 徐勇前等 ${ }^{[39]}$ 报道了一种 $\mathrm{Ga}(\mathrm{III})$ 自组装的荧光传 感器 27. 其能够通过苂光增强实现 ATP 的选择性识别. 此外, 该传感器能够应用于检测 ATP 相关酶的活性. 其 机理为 ATP 的腺苷部分和有机配体之间存在 $\pi-\pi$ 堆积作 用, 其对 ATP选择性识别起到关键作用. 传感器 27 被成 功应用于细胞内 ATP 的检测. 该研究工作是首次通过 $\mathrm{Ga}(\mathrm{III})$ 实现 ATP 检测的苂光传感器. 最近, 肖猱等 ${ }^{[40]}$ 报 道了基于结构简单的䒺酚苂光团的金属 $\mathrm{Ga}(\mathrm{III}) / \mathrm{ATP}$ 苂 光传感器 28. 28-Ga(III)配合物在水溶液中对 ATP 和 ADP 表现出高的选择性和灵敏性. 通过生物实验研究 发现, 该研究结果可以用于细胞内和斑马鱼中 ATP 和 ADP 的检测.

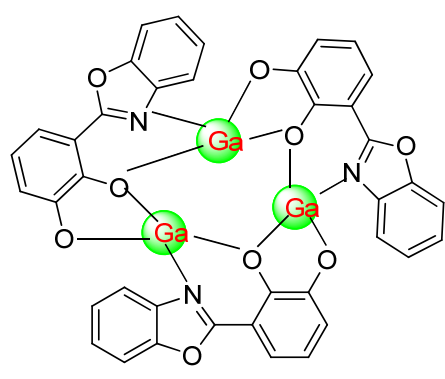

27

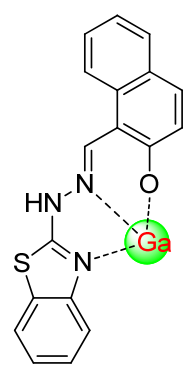

28
金属 $\operatorname{Co}(\mathrm{II})$ 三结点配合物 29, 30 用于在水溶液中 ATP 检测被段春迎等 ${ }^{[41]}$ 报道. 其结构由发色基团和氢键 结合位点组成, 基于 $\mathrm{Co}(\mathrm{II})$ 的螺旋形三角形结通过 $\mathrm{NH}_{4} \mathrm{PF}_{6}$ 加入到甲醇溶液的配体和 $\mathrm{Co}\left(\mathrm{NO}_{3}\right)_{2} \cdot 6 \mathrm{H}_{2} \mathrm{O}$ 中所 制的. 这些 $\mathrm{Co}(\mathrm{II})$ 为基础的螺旋结点结构 29 和 30 中加 入 ATP 时表现出几乎相同的 UV-vis 光谱变化, 在 300 $\mathrm{nm}$ 处吸收峰增加和 $380 \mathrm{~nm}$ 处出现明显的吸收峰降低. 其与 ATP 的结合比为 $1: 1$, 结合常数分别为 $(2.55 \pm$ $0.03)$ 和(3.86 \pm 0.03$)$.

2013 年，唐瑜等 ${ }^{[42]}$ 报道了一种基于 $\mathrm{Eu}$ 配合物的苂 光传感器 31 , 在 $\mathrm{pH}$ 为 6.8 的条件下, 能够有效地从

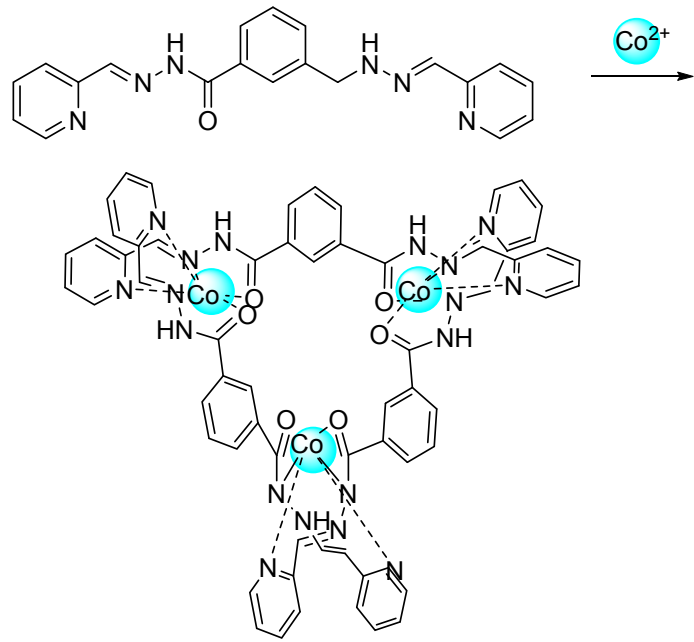

29<smiles>CN(C)c1cccc2c(S(=O)(=O)Nc3cc(CN/N=C/c4ccccn4)cc(C(=O)N/N=C/c4ccccn4)c3)cccc12</smiles><smiles>CN(C)c1ccc(S(N)(=O)=O)c2ccccc12</smiles>

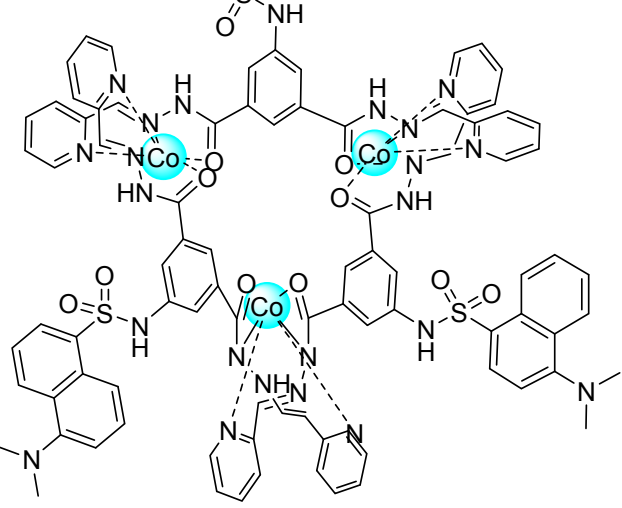

30

图式 6 传感器 29 和 30 的结构图 Scheme 6 Structures of 29 and $\mathbf{3 0}$

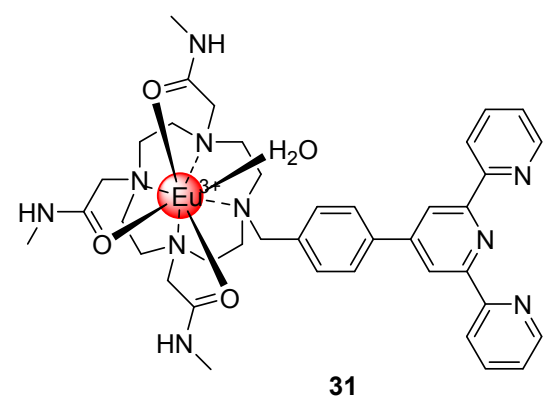


ADP 和 AMP 的混合水溶液中选择性识别 ATP. 该结构 具有长的荧光寿命, 具有潜在应用于生物体系监测 ATP 水平的可能性, 可以完全消除其它分子的背景苂光信号 干扰. 该工作为高选择性和专一性生物分子镧系元素苂 光传感器提供新的方法. Pierre 等 ${ }^{[43]}$ 报道了一种发冷光 的核苷酸苂光传感器 32, 其结构以稀土元素 $\mathrm{Tb}$ 为基础, 具有很高的热力学稳定性和动力学稳定性. 在中性 $\mathrm{pH}$ 条件下缓冲溶液中能够检测三磷酸腺苷. 另外, 可以监 测 ATP 转化为 ADP 或 AMP 的酶反应. 传感器能够监测 mmol 浓度的 ATP, 其监测水平与细胞内的环境相一致. 其检测机理为, 在 ATP 存在的条件下, 嘌呤基团与传感 器结构中的菲啶结构堆叠产生光电子转移. 该研究结果 可以应用于高通量篎选和激酶抑制的动力学研究.

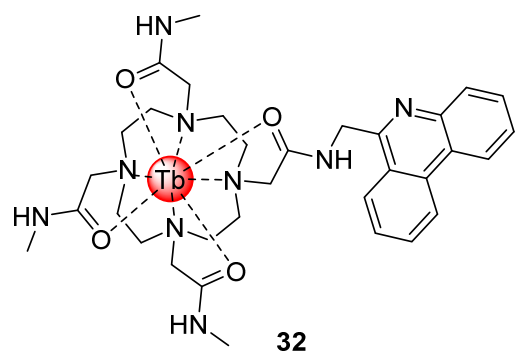

最近, Parker 等 ${ }^{[44]}$ 报道了一系列稀土元素 $\mathrm{Eu}$ 配合物 33 35, 其结构中包含氨甲基吡啶对 $\mathrm{Zn}(\mathrm{II})$ 和三种核苷 酸 ATP, ADP, AMP 的结合能力不同, 当体系中加入 ATP 之后, 其增强的苂光发射强度被观察到. 配合物产生循 环感应的极化发射效应, 其 ATP 和 ADP 的信号相反. 对 于 $\mathrm{ADP} / \mathrm{ATP}$ (或 AMP/ATP)的比例可以通过荧光信号的 监测被评估. 进一步通过圆二偏振发光谱(CPL), 可以 区分核苷酸和在溶液中监测 ADP 和 ATP 的比例. 核苷 酸与 $[\mathbf{E u 3 1}] \cdot \mathrm{Zn}^{2+}$ 和 $[\mathbf{E u 3 2}] \cdot \mathrm{Zn}^{2+}$ 导致手性相反的 CPL 谱, 可以实现 $\mathrm{mmol}$ 浓度核苷酸的监测.
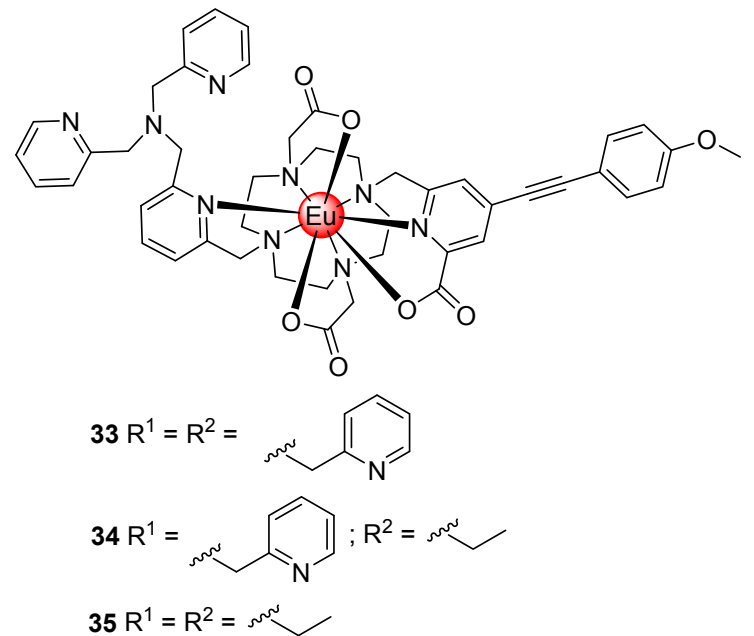

最近, Butler 等 ${ }^{[45]}$ 报道了一种基于阳离子 $\mathrm{Eu}$ 配合物 检测细胞内线粒体 ATP 浓度的苂光传感器 36 . 在涉及
细胞内新陈代谢和酶反应过程的研究长久以来缺乏灵 敏性的苂光成像传感器，实现快速确定和区分细胞内 ATP 的浓度. 该工作运用镧系元素化合物能够可视化观 察到线粒体中 ATP 浓度的变化. Eu 配合物对 ATP 表现 出强的结合能力, 在高竞争性离子存在的水溶液环境中 $\left(\mathrm{Mg}^{2+}, \mathrm{ADP}, \mathrm{GTP}, \mathrm{UTP}\right.$ 和人类血清蛋白)能够实现对 ATP, 快速, 长寿命的荧光信号. 这种 $\mathrm{Eu}(\mathrm{III})$ 配合物对 ATP 生理浓度范围内 $(1 \sim 5 \mathrm{mmol} / \mathrm{L})$ 的响应成线性关系. 另外, 可以监测三磷酸腺苷酶催化水解的 ATP 到 ADP 的过程. 传感器 36 能够在 NIH-3T3 细胞中具有良好的 通透性，在线粒体中表现出高的选择性。该策略采用 Eu(III)的配合物在时间和空间上监测 ATP 的动力学过 程，通过结构的优化实现苂光强度的比率型变化.

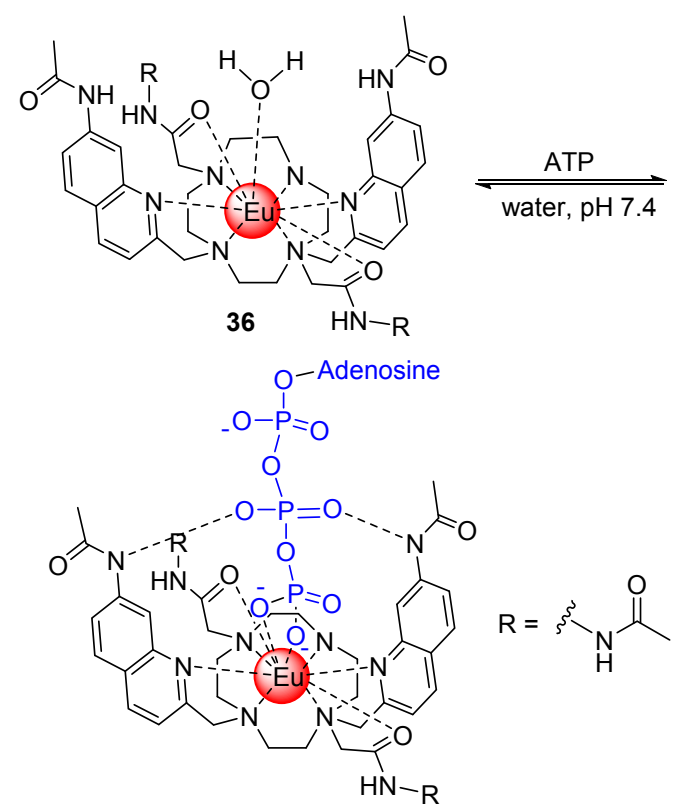

图式 7 传感器 36 对 ATP 的结合模式和识别机理 Scheme 7 Signalling mechanism and proposed binding mode between chemosensor $\mathbf{3 6}$ and ATP

\section{3 静电或氢键作用的化学传感器}

基于静电或氢键作用的 ATP 苂光传感器, 通常使用 酰胺、吡咯、咪唑鎓盐和胍盐等 ${ }^{[46]}$. 该类荧光传感器对 ATP 的选择性识别和检测具有重要意义. 下面对以氢键 或电荷作用的 ATP 苂光传感器做一总结.

2009 年, Joon 等 ${ }^{[47]}$ 报道了一种钳状的基于萠和咪唑 鎓盐, 由苯环桥连的 ATP 苂光传感器 37 . 当 ATP 存在的 情况下, 通过苂光信号可以观察到. 其原因为腺苷萠之 间的 $\pi-\pi$ 堆积相互作用. 另一方面，四种其他的三磷酸 核苷如 GTP、CTP、UTP、TTP 只能与探针中稳定的的 萠一萠堆积二聚体相互作用，使得激态分子荧光猝灭. 当传感器结合 ATP之后，从单体到激态分子的苂光强度 比率 $I_{345} / I_{487}$ 比结合 ADP 或 AMP 大得多. 如此大的差距 
使得可以将 ATP 从 ADP 和 AMP 中区分开来. 传感器中 萠-腺嘌呤-萠形成独特的三明治堆积结构作为性能良 好的 ATP 苂光传感器为 ATP 相关疾病的研究提供一种 重要工具.

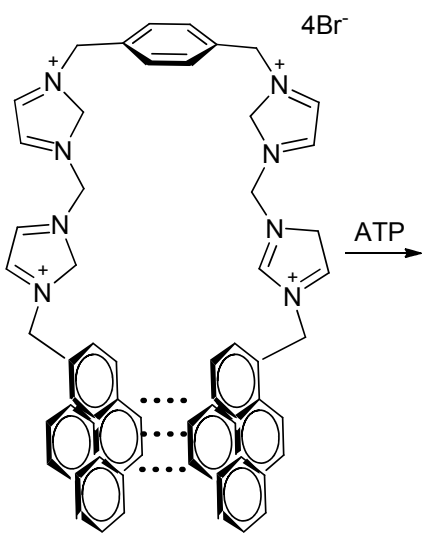

37

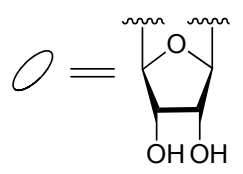

图式 8 传感器 37 与 ATP 的结合模式

Scheme 8 Proposed binding mode between chemosensor 37 with ATP

最近, Anzenbacher 等 ${ }^{[48]}$ 利用咪唑鎓盐肼取代的葱 结构作为 ATP 选择性传感器 38. 在 Tris 缓冲溶液中, 传 感器 38 对 ATP 具有显著的选择性，其苂光强度增加 35 倍. 另外, 除了对 ADP 显示出 8 倍苂光增强外, 其对 AMP、GTP、CTP、UTP 等核苷酸磷酸盐均未产生明显 的荧光增强现象. 二取代葱对 ATP 强的选择性归因于其 与 ATP 的静电吸引和 $\pi-\pi$ 相互作用, 导致强的结合能力. 更进一步研究发现，其它一些简单阴离子如 $\mathrm{F}^{-} 、 \mathrm{Cl}^{-}$、 $\mathrm{AcO}^{-} 、 \mathrm{H}_{2} \mathrm{PO}_{4}^{-} 、 \mathrm{HPO}_{4}^{2-}$ 等也不产生荧光增强现象. 一 般情况下, 细胞内 ATP 的浓度维持在 $1 \sim 10 \mathrm{mmol} \cdot \mathrm{L}^{-1}$ 范围内, ATP/ADP 的正常比例约为 1000. 该传感器可以 检测的浓度范围为 $1 \sim 12 \mathrm{mmol} \cdot \mathrm{L}^{-1}$, 适合于正常细胞中 ATP 的检测, ADP 的存在没有干扰. 遗憾的是, 该工作 没有细胞水平上 ATP 检测的相关研究.

自从 2001 年聚集诱导发光现象被唐本忠课题组发 现以来, 关于 AIE 效应的有机化合物被不断报道 ${ }^{[49-51]}$. 特别是四苯乙烯(TPE)类化合物的聚集效应最为明显, 且合成方便、结构容易修饰的特点引起人们的高度关注.

2014 年, 杨国强等 ${ }^{[52]}$ 报道了一种结构新颖的基于 三联硼的 2-萠-1-(2,4,6-三异丙基)硼烷(DPTB) ATP 荧光 传感器 39. 该传感器能够在体内和体外监测 ATP 的水 平, 且对 ATP 表现出比 ADP 和 AMP 以及生物中其它 离子高的专一性. 通过荧光显微方法研究发现, 其被

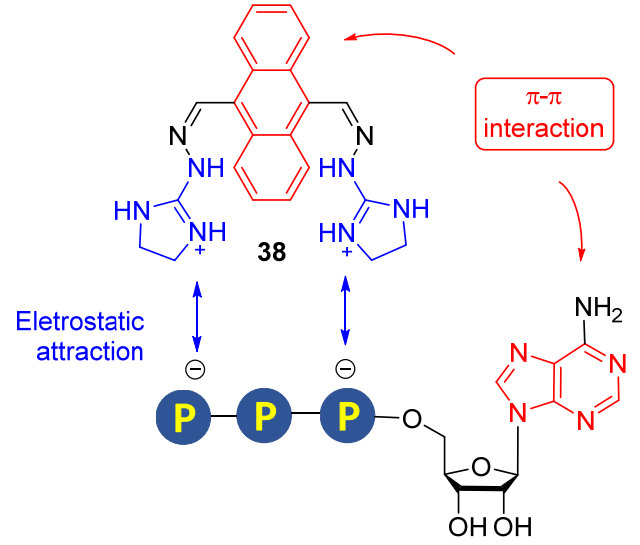

成功应用于 NIH/3T3 细胞中的 ATP 成像研究. 在识别的 过程中, 不同 ATP 浓度的大小在细胞质和细胞膜中能 够清晰可见. 其识别过程为 ATP 诱导使聚集状态出现, 提高了传感器 39 的光稳定性. 通过生物实验研究发现, 化合物表现出低的细胞毒性，良好的细胞穿透性，以及 良好的可分散性，对 ATP 在细胞中的分散表现出良好的 成像性能, 且能够跟踪细胞器中 ATP 的分布. 该传感器 成功应用于 $\mathrm{NIH} / 3 \mathrm{~T} 3$ 细胞中 ATP 水平的测定.

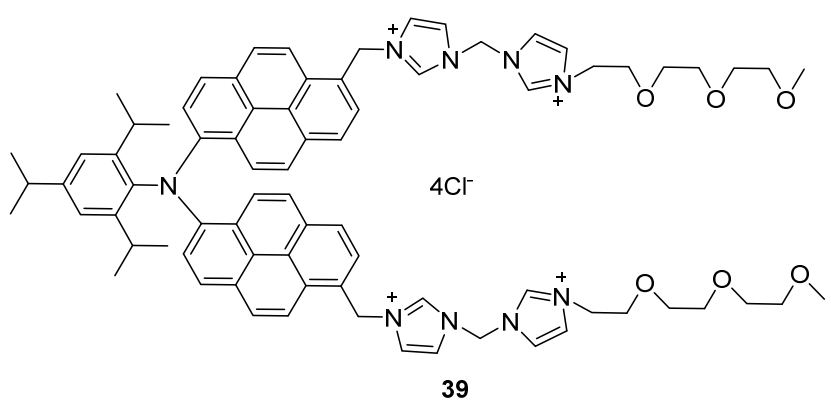

2016 年，李立东等 ${ }^{[53]}$ 报道了一种自组装的荧光有 机纳米颗粒. 可以用于 ATP 和 $\mathrm{Fe}(\mathrm{III})$ 的识别传感器 $\mathbf{4 0}$. 近年来, 荧光有机纳米颗粒在分子识别和生物成像方面 吸引了人们的广泛关注，主要归因于低的毒性，灵活的 制备方法, 以及表面可以功能化. 在该研究工作中, 在 水溶液中通过自组装得到苂光有机纳米颗粒, 其表现出 很微弱的苂光. 当传感器所在的体系中加入 ATP 之后, 荧光纳米颗粒很快聚集形成且表现出很强的荧光, 其原 因为强的静电作用和 $\pi-\pi$ 堆积作用. 这种荧光纳米颗粒 对 $\mathrm{Fe}(\mathrm{III})$ 表现出高的选择性和灵敏性, 其检测限可以达 到 $0.1 \mathrm{nmol} / \mathrm{L}$. 除此之外, 当用传感器 40 培养 Hela 细胞 之后,细胞内内生的 ATP 表现出极其优异的苂光成像效 果.

最近，曹利平等 ${ }^{[54]}$ 采用 $\mathrm{S}_{\mathrm{N}} 2$ 反应合成了两种含有四 苯乙烯和双吡啶基的阳离子型环蕃结构 41，42. 作者通 过单晶衍射分析发现, 42 的结构比 41 具有更大的空腔. 


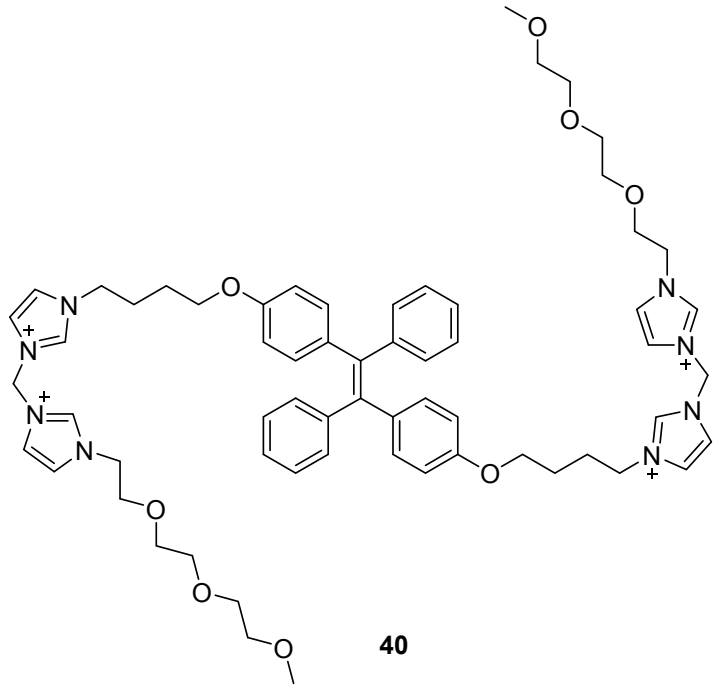

在水溶性中, 两种环蕃对氨基酸表现出苂光淬灭效果, 通过静电作用和 $\pi-\pi$ 相互作用形成 $1: 1$ 的主客体化合 物, 其结合常数达到 $10^{8} \sim 10^{9} \mathrm{~L} \cdot \mathrm{mol}^{-1}$. 研究发现, 化合 物 41 对色氨酸和 ATP 表现出高的选择性识别能力, 其 可能的原因为合适的空腔大小和良好的水溶性. 作者设 想将这种阳离子环蕃与其它功能基团连接, 并对其空腔 结构进行优化, 合成更多的生物探针来检测生物分析物 (如 DNA 等), 也是超分子构建分子结的一种途径. 2017 年，王建国等 ${ }^{[55]}$ 采用类似的识别基团合成了 AIE 苂光传 感器 43, 用于在水溶液中 ATP 的检测.
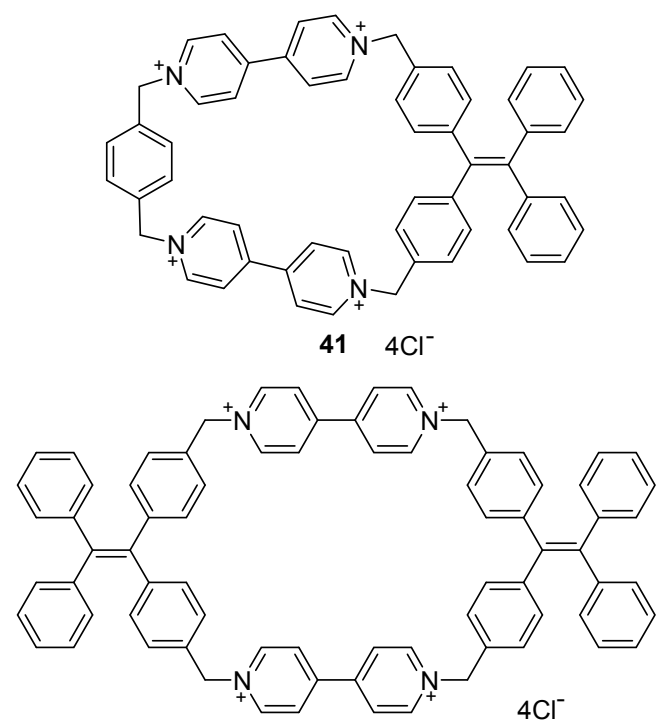

42

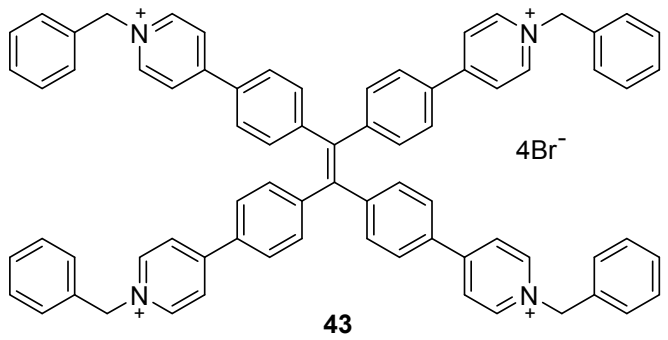

2012 年, Shinkai 等 ${ }^{[56]}$ 报道了一种基于四苯乙烯胍 盐的 ATP 的生物苂光传感器 44. 其对 ATP 表现出非线 性的苂光响应，同时伴随着 ATP 诱导的四苯乙烯结构自 组装导致 AIE 效应的发生. 基于 AIE 效应的探针模型的 设计为其它生物小分子的检测提供更多的机遇. 生物试 验研究发现，传感器 44 可以应用于检测 DNA 序列，其 对嘌呤环的结合能力强于嘧啶环. 另外, 其对不同核酸 结构的苂光响应顺序依次为: GTP $>$ ATP $>$ CTP $>$ UTP. 该结果为其它生物分子负载着酸盐和硫酸阴离子基团 的相关传感器设计提供参考信息. 该工作也为基于 AIE 效应的生物苂光分子和分子自组装的相互结合构建新 型传感器打开新的窗口.

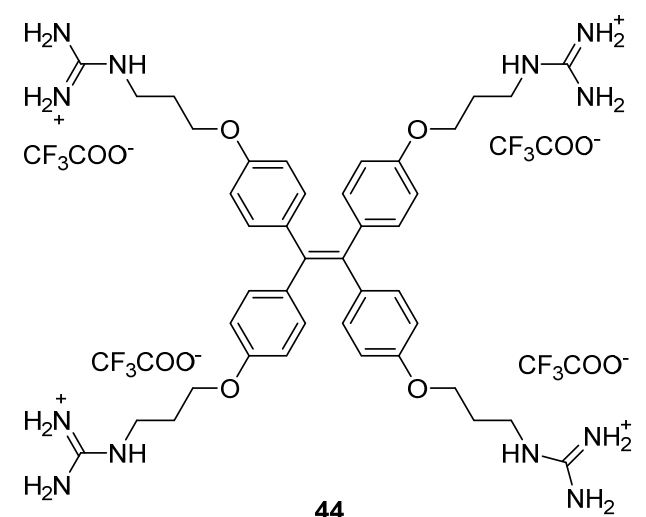

最近, Schmuck 等 ${ }^{[57]}$ 报道了一种基于缩氨酸的为苂 光团的 ATP 苂光传感器 45. 其结构中䒺二甲酰亚胺作 为苂光基团，两个对称的缩氨酸结构的臂和两个胍盐， 形成剪刀形状的阴离子键合位点，实现对三磷酸核苷的 检测. 当溶液中存在三磷酸腺苷时, 其表现出 turn-on 型 的苂光响应. 传感器 45 与 ATP 的结合常数为 $2.2 \times 10^{5}$ $\mathrm{L} \cdot \mathrm{mol}^{-1}$, Job plots 研究发现其结合比为 $1 ： 1$. 此外, 细 胞试验研究发现，其能够用于细胞内 ATP 的检测. 该研 究结果可以作为生物分析工具用于细胞内 ATP 的荧光 成像研究.

2017 年，李春艳等 ${ }^{[58]}$ 报道了一种能够在线粒体中 识别 ATP 的荧光传感器 46. 在结构设计中, 分别采用罗 丹明、二亚乙基三胺和三苯基膦作为苂光基团，反应位 点和线粒体靶向基团. 该传感器结构是首次合成且被用 于线粒体中 ATP 的识别. 其对 ATP 表现出高的灵敏性, 并伴随着 81 倍的苂光发射的增强，其检测范围为 $0.1 \sim$ $10 \mathrm{mmol} \cdot \mathrm{L}^{-1}$. 更进一步研究发现，探针对 ATP 具有极 高的选择性，其超过其它生物阴离子如 ADP, AMP, GTP, CTP 和 UTP, 其原因为氢键和 $\pi-\pi$ 堆积作用的协同效应. 该传感器能够作为线粒体专一性标记、实时用于线粒体 中 ATP 变化检测的工具.

2014 年, 他们课题组 ${ }^{[59]}$ 报道了一种以萘二甲酰亚 胺-罗丹明为荧光团的比率型荧光传感器 47 , 可以用于 


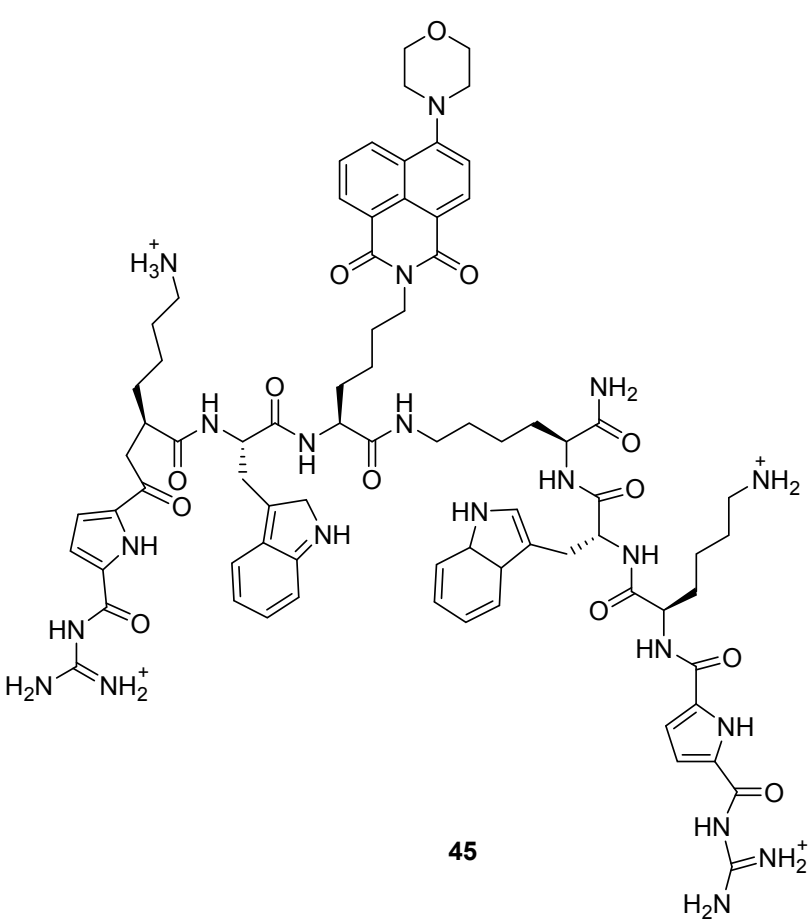

溶液和细胞中 ATP 的检测. 该探针表现出不寻常的性 能, 其对 ATP 表现出高选择性超过其它任何有机磷酸阴 离子. 其检测机理为多磷酸链和核苷酸基团的同步作 用, 当传感器所在溶液中加入 ATP 之后, 形成罗丹明ATP 配合物使得菜酰胺环打开, 最终结果为能量从荎二 甲酰亚胺转移到罗丹明部分. 该工作是首次利用苂光共 振能量转移(FRET)机理荧光选择性检测 ATP 的罗丹明 化合物，该策略为其它磷酸根阴离子荧光传感器的设计 提供思路.

2018 年，马会民等 ${ }^{[60]}$ 报道了一种基于罗丹明结构 的双功能型荧光传感器 48 , 其能够同时对硝基还原酶 (NTR), ATP 和二者混合物(NTR+ATP)表现出不同的荧 光响应信号. 该传感器能够检测两种低氧敏感的 NTR,<smiles>CCN(CC)c1ccc2c(c1)Oc1cc(N(CC)CC)ccc1C21C(=O)c2ccccc2N1CCNCCNC(=O)CCCP(c1ccccc1)(c1ccccc1)(c1ccccc1)c1ccccc1</smiles>

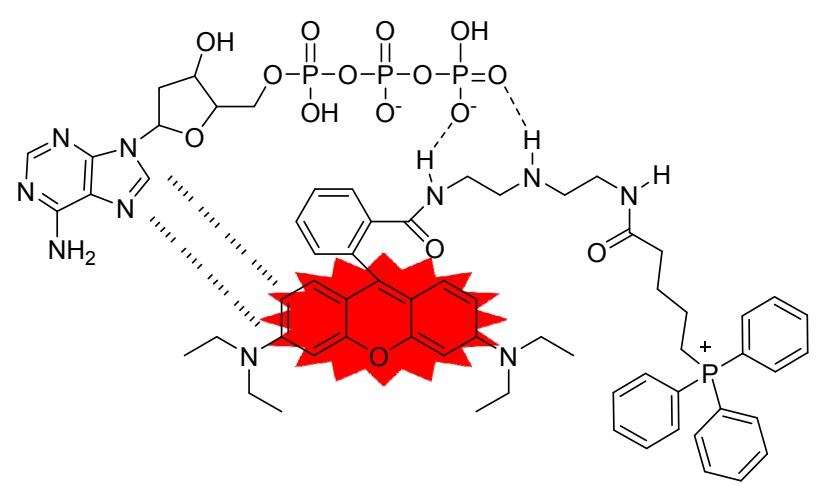

图式 9 传感器 $\mathbf{4 6}$ 与 ATP 的作用机理

Scheme 9 Proposed response mechanism of chemosensor 46 to ATP

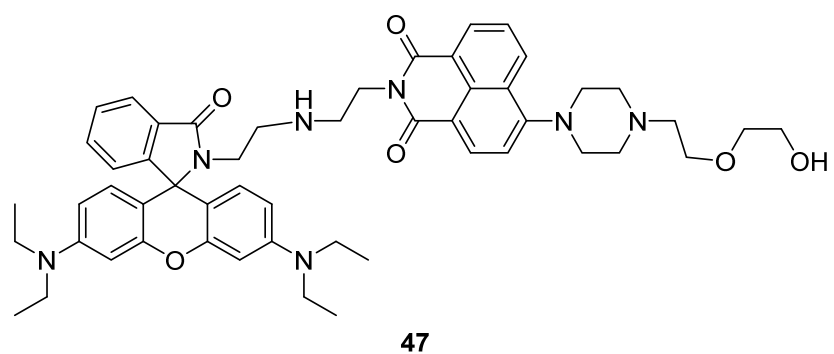

ATP 和 ATP + NIR. 相关生物试验研究发现, 其在 Hela 细胞中能够实现检测. 这是第一次发现细胞内 NTR 和 ATP 产生相反低氧过程的变化趋势. 该研究结果得出 ATP 与 NTR 结合可以用于低含氧量状态细胞的准确诊 断.

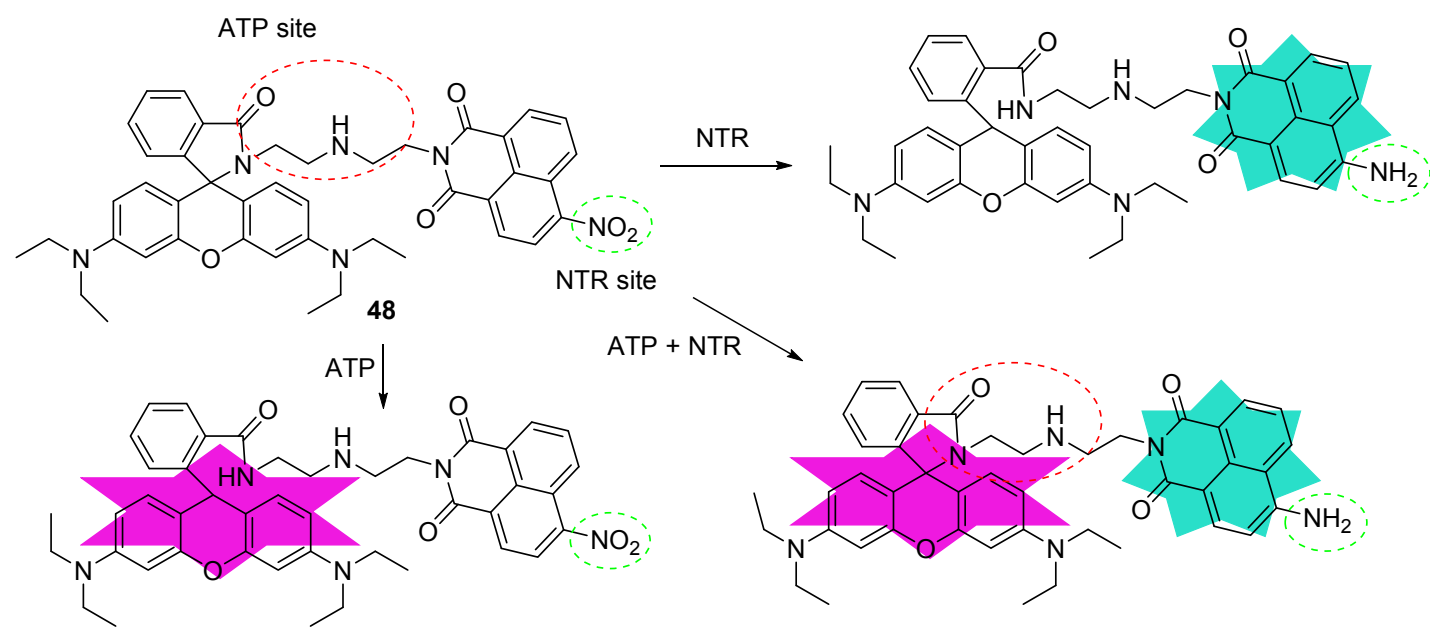

图式 10 传感器 48 的结构与 NTR, ATP 和 NTR/ATP 的反应机理

Scheme 10 Structure of the chemosensor 48 and its reaction with NTR, ATP and NTR/ATP 
袁林等 ${ }^{[61]}$ 报道了一种多位点结合的 ATP 荧光传感 器 49, 其能够在细胞内快速地选择性响应 ATP. 其响应 机理为在 ATP 存在的情况下, 通过硼酸和核糖的共价键 作用，氧杂葱和腺嘌呤之间的 $\pi-\pi$ 作用以及氨基和磷酸 基之间的静电作用实现苂光信号的转变, 产生强的苂光 信号. 细胞成像试验研究表明, 传感器能够在线粒体中 定位，且具有很好的生物相容性及细胞膜穿透性. 在传 感器的作用下，作者成功观察到在细胞处于能量不足的 情况下, 线粒体中 ATP 含量的减少以及细胞凋亡初期线 粒体中 ATP 浓度的增加现象. 该传感器为研究 ATP 相关 的生理过程提供一种有力的工具.
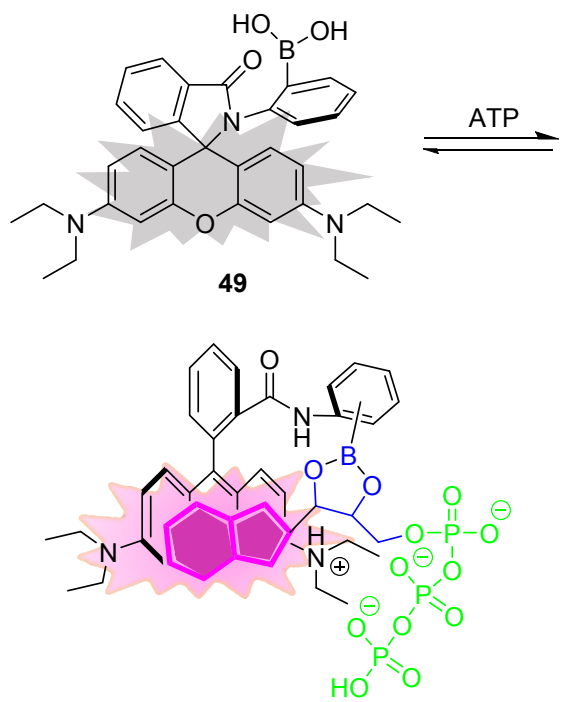

图式 11 传感器 49 识别 ATP 的机理

Scheme 11 Proposed mechanism of chemosensor 49 for sensing ATP

2017 年, Sahoo 等 ${ }^{[62]}$ 基于荎和罗丹明结构合成了一 种在水溶液检测 cAMP (3,5-环单磷酸腺苷)和 Aps (ATP, ADP, AMP) 的传感器 $\mathbf{5 0}$. 该传感器对 cAMP 和 ATP 等表 现出高的灵敏性和选择性, 可通过比色和荧光检测技术 实现对其检测. 其对 cAMP 检测限在 mmol 范围内, 可 用于诊断学和细胞成像等相关研究. 此外, 该传感器可 以通过比色或荧光实现哺乳动物血液细胞和其它样品 中 cAMP 的检测. 该研究结果为人类所需生物制剂的篎 选提供可能的工具.

2018 年, Yoon 等 ${ }^{[63]}$ 基于罗丹明衍生物开发了一种 ATP 荧光传感器 51, 其检测机理为氢键作用. 传感器 $\mathbf{5 1}$ 所在的溶液中加入 $5 \mathrm{mmol} \cdot \mathrm{L}^{-1}$ ATP 之后, 其表现出 65 倍的苂光强度的增强, 并伴随着颜色从无色到粉红色的 变化. 在 ATP 存在的情况下, 传感器 52 同样表现出明显 的颜色和苂光改变. 该研究结果表明, 这种类型的苂光 传感器对 ATP 具有很高的专一性. 其荧光发射的增强和 肉眼观察到的颜色改变归因于螺环的打开, 其所适用的 $\mathrm{pH}$ 范围为 4.0 7.4. 该研究结果发现吸电子基团能够降

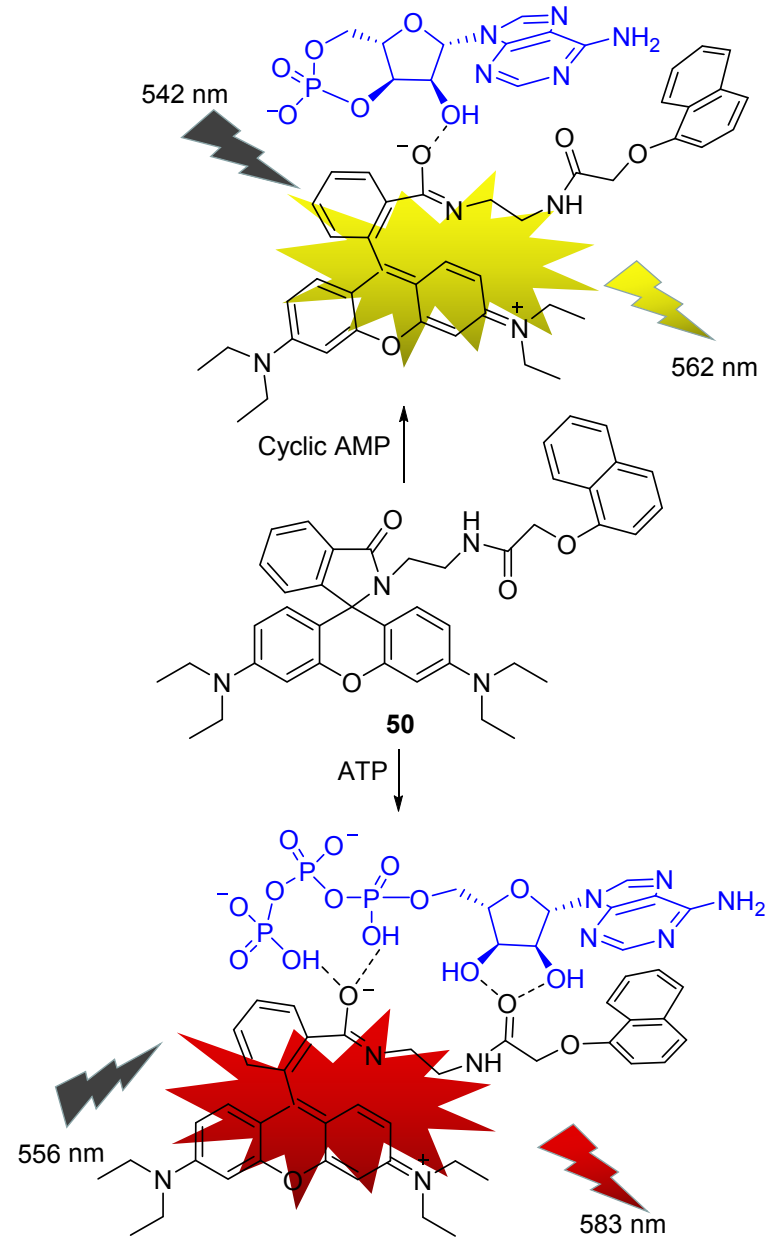

图式 12 加入 $\mathrm{CAMP}$ 和 ATP 引起传感器 $\mathbf{5 0}$ 荧光的变化 Scheme 12 Fluorescence changes of $\mathbf{5 0}$ upon addition of cAMP and ATP

低 ATP 的结合能力. 通过细胞试验研究发现, 传感器 $\mathbf{5 2}$ 在 Hela 细胞中具有良好的细胞渗透性, 可以用于细胞 线粒体标记.
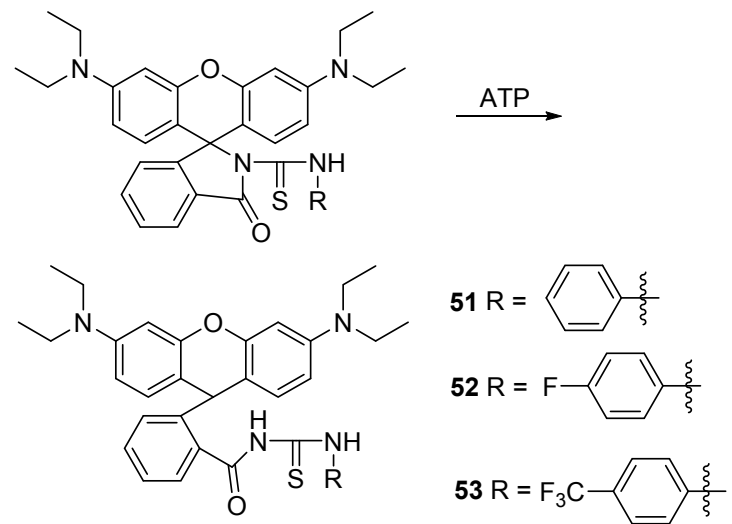

图式 13 传感器 51,52 和 53 的结构图

Scheme 13 Structures of chemosensors 51, 52 and 53

2017 年, 江云宝等 ${ }^{[64]}$ 设计合成一种苂光传感器 54, 当形成 $\mathrm{H}$-聚集态时，其荧光发射被淬灭．当体系中加入 
相关生物分子或离子时, 其聚集态转变到另一种形式, 产生强的苂光信号, 从而达到检测的目的. 聚集的荧光 染料提供多结合位点, 与识别物进行识别. 传感器 54 具 有低背景干扰, 强的结合能力和强的苂光信号, 具有高 的识别能力. 且随着信号的放大, 其选择性也有所提高. 作者在所设计的分子结构中, 采用花菁染料结构包含两 个喼酸基官能团用于 ATP 苂光识别, 硼酸基团与 ATP 的顺式二醇部分结合. 在该研究工作中, 引入阳离子表 面活性剂十二烷基三甲基溴化铵(DTAB)是非常重要的 因素. 在 ATP 存在的情况下, 三种物质形成囊泡, 苂光 染料被很好地分散和保护, 因此苂光发生显著增强, 其 能够在水溶液中高灵敏度和高选择性检测 ATP.
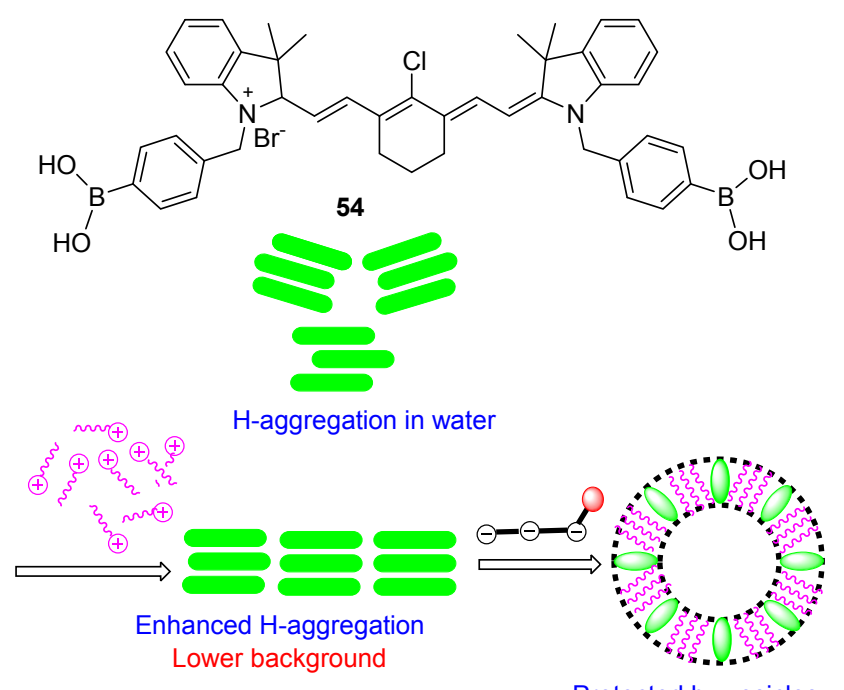

Protected by vesicles Stronger output signal

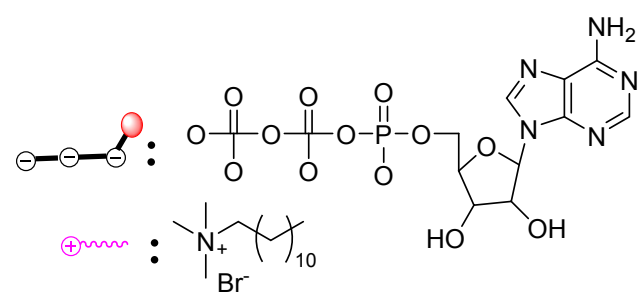

图式 14 近红外传感器 54 的化学结构及 ATP 识别示意图 Scheme 14 Chemical structures of chemosensor 54; schematic diagram of sensing ATP by near-IR fluorescent chemosensor

\section{4 总结与展望}

最近几年, 苂光传感器在核酸类物质的检测方面得 到了迅速的发展, 对相关疾病的研究起到非常重要的作 用. 综述了可以用于 ATP 检测的有机小分子荧光传感 器, 其荧光基团主要为 $\mathrm{Zn}(\mathrm{II})$ 配合物、其它金属离子配 合物和四苯乙烯及罗丹明等有机小分子, 并探讨了传感 器的设计原理、响应机制和生物应用. 通过综述发现, DPA-Zn(II)结构单元与各种荧光发光基团连接的荧光传 感器已被广泛用于 ATP 的检测, 但该类传感器大多数存
在水溶性较差的缺点, 还不能完全满足生物体内环境的 检测要求. 虽然本文提到的一部分传感器对 ATP 具有高 灵敏度和专一性，但是仍然需要科技工作者研发更适合 于生命体内应用的新传感器. 在性能优异的 ATP 传感器 设计中, 主要考虑生物和化学两个方面因素, 化学方面 需要考虑 ATP 结合位点 [目前大多数以 Zn(II)配合物、氢 键或静电作用为主], 生物方面需要考虑选择性, 灵敏 性、良好的溶解性以及生物环境物质的低干扰. 目前, 关于近红外 ATP 苂光传感器很少有报道, 该类传感器具 有低的背景干扰和较深的组织渗透性, 对生物体内 ATP 的成像检测具有很大的潜在应用价值. 综上所述, 开发 性能更加优异的用于细胞和生命体中研究相关生理和 病理功能的 ATP 苂光传感器仍然是生命科学和医学领 域的热点和难点.

\section{References}

[1] Knowles, J. R. Annu. Rev. Biochem. 1980,49,877.

[2] Higgins, C. F.; Hiles, I. D.; Salmond, G. P. C.; Gill, D. R.; Downie, J. A.; Evans, I. J.; Holland, I. B.; Gray, L.; Buckel, S. D.; Bell, A. W.; Hermodson, M. A. Nature 1986, 323, 448.

[3] Mishra, N. S.; Tuteja, R.; Tuteja, N. Arch. Biochem. Biophys. 2006, 452,55 .

[4] Ghosh, A.; Shrivastav, A.; Jose, D. A.; Mishra, S. K.; Chandrakanth, C. K.; Mishra, S.; Das, A. Anal. Chem. 2008, 80, 5312.

[5] Beyeh, N. K.; Diez, I.; Taimoory, S. M.; Meister, D.; Feig, A. I.; Trant, J. F.; Ras, R. H. A.; Rissanen, K. Chem. Sci. 2018, 9, 1358.

[6] Gao, M.; Yu, F.; Lv, C.; Choo, J.; Chen, L. Chem. Soc. Rev. 2017, 46, 2237.

[7] Umezawa, K.; Yoshida, M.; Kamiya, M.; Yamasoba, T.; Urano, Y. Nat. Chem. 2017, 9, 279.

[8] You, L.; Zha, D.; Anslyn, E. V. Chem. Rev. 2015, 115, 7840.

[9] Buccella, D.; Horowitz, J. A.; Lippard, S. J. J. Am. Chem. Soc. 2011, 133, 4101.

[10] Zhou, Y.; Xu, Z.; Yoon, J. Chem. Soc. Rev. 2011, 40, 2222.

[11] Weitz, E. A.; Chang, J. Y.; Rosenfield, A. H.; Pierre, V. C. J. Am. Chem. Soc. 2012, 134, 16099.

[12] Lu, B.; Meng, S.; Feng, Y. Chin. J. Org. Chem. 2018, 38, 350 (in Chinese). (卢博为, 孟舒献, 冯亚青, 有机化学, 2018, 38, 350.)

[13] Qu, W.; Fang, H.; Huang, Q.; Zhang, Y.; Lin, Q.; Yao, H.; Wei, T. Chin. J. Org. Chem. 2019, 39, 1226 (in Chinese). (曲文娟，房虎，黄青，张有明，林奇，姚虹，魏太保，有机化学， 2019, 39, 1126.)

[14] Li, J.; Chen, L.; Du, L.; Li, M. Chem. Soc. Rev. 2013, 42, 662.

[15] Fan, Y.; Wang, S.; Zhang, F. Angew. Chem. Int. Ed. 2019, 58, 13208.

[16] Li, M.; Wang, Y.; Liu, G.; Lü, H.; Xing, G. Chin. J. Org. Chem. 2017, 37, 356 (in Chinese).

(李美含, 王宇童, 刘广建, 吕海娟, 邢国文, 有机化学, 2017, 37, 356.)

[17] Kurishita, Y. ; Kohira, T.; Ojida, A.; Hamachi, I. J. Am. Chem. Soc. 2010, 132, 13290.

[18] Wu, Y.; Wen, J.; Li, H.; Sun, S.; Xu, Y. Chin. Chem. Lett. 2017, 28, 1916.

[19] Ojida, A.; Mito-oka, Y.; Sada, K.; Hamachi, I. J. Am. Chem. Soc. 2004, 126, 2454.

[20] Yamaguchi, S.; Yoshimura, I.; Kohira, T.; Tamaru, S.; Hamachi, I J. Am. Chem. Soc. 2005, 127, 11835.

[21] Ojida, A.; Miyahara, Y.; Wongkongkatep, J.; Tamaru, S.; Sada, K.; Hamachi, T. Chem. Asian J. 2006, 1, 555. 
[22] Ojida, A.; Nonaka, H.; Miyahara, Y.; Tamaru, S.; Sada, K.; Hamachi, I. Angew. Chem. Int. Ed. 2006, 45, 5518.

[23] Jose, D. A.; Mishra, S.; Ghosh, A.; Shrivastav, A.; Mishra, S. K.; Das, A. Org. Lett. 2007, 9, 1979.

[24] Mahato, P.; Ghosh, A.; Mishra, S. K.; Shrivastav, A.; Mishra, S.; Das, A. Inorg. Chem. 2011, 50, 4162.

[25] Duodu, E.; Kraskouskaya, D.; Gomez-Biagi, R. F.; Gunning, P. T. Analyst 2016, 141, 820.

[26] Butler, S. J. Chem. Eur. J. 2014, 20, 15768.

[27] Minami, T.; Emami, F.; Nishiyabu, R.; Kubo, Y.; Anzenbacher, P. Jr. Chem. Commun. 2016, 52, 7838 .

[28] Rao, A. S.; Kim, D.; Nam, H.; Jo, H.; Kim, K. H.; Ban, C.; Ahn, K. H. Chem. Commun. 2012, 48, 3206.

[29] Zhang, M.; Ma, W. J.; He, C.T.; Jiang, L.; Lu, T. B. Inorg. Chem. 2013, 52, 4873

[30] Fang, W.; Liu, C.; Yu, F.; Liu, Y.; Li, Z.; Chen, L.; Bao, X.; Tu, T. ACS. Appl. Mater. Interfaces 2016, 8, 20583.

[31] Moro, A. J.; Cywinski, P. J.; Korsten, S.; Mohr, G. J. Chem. Commun. 2010, 46, 1085.

[32] Xu, Z.; Spring, D. R.;Yoon, J. Chem. Asian. J. 2011, 6, 2114.

[33] Xu, Q.; Lv, H.; Lv, Z.; Liu, M.; Li, Y.; Wang, X.; Zhang, Y.; Xing, G. RSC Adv. 2014, 4, 47788 .

[34] Strianese, M.; Milione, S.; Maranzana, A.; Grassi, A.; Pellecchia, C. Chem. Commun. 2012, 48, 11419.

[35] Patra, C.; Bhanja, A. K.; Mahapatra, A.; Mishra, S.; Saha, K. D.; Sinha, C. RSC Adv. 2016, 6, 76505.

[36] Kumari, N.; Zelder, F. Chem. Commun. 2015, 51, 17170.

[37] Kataev, E.; Arnold, R.; Rüffer, T.; Lang, H. Inorg. Chem. 2012, 51, 7948.

[38] Gao, Y. G.; Tang, Q.; Shi, Y. D.; Zhang, Y.; Lu, Z. L. Talanta 2016, $152,438$.

[39] Xiao, L.; Sun, S.; Pei, Z.; Pei, Y.; Pang, Y.; Xu, Y. Biosens. Bioelectron. 2015, 65, 166.

[40] Zhang, X.; Jiang, Y.; Xiao, N. Chem. Commun. 2018, 54, 12812.

[41] Wu, H.; He, C.; Lin, Z.; Liu, Y.; Duan, C. Inorg. Chem. 2009, 48, 408.

[42] Liu, X.; Xu, J.; Lv, Y.; Wu, W.; Liu, W.; Tang, Y. Dalton. Trans. 2013, 42, 9840 .

[43] Weitz, E. A.; Chang, J. Y.; Rosenfield, A. H.; Pierre, V. C. J. Am. Chem. Soc. 2012, 134, 16099.

[44] Shuvaev, S.; Fox, M. A.; Parker, D. Angew. Chem., Int. Ed. 2018 , 57,7488 .
[45] Mailhot, R.; Traviss-Pollard, T.; Pal, R.; Butler, S. J. Chem. Eur. J. 2018, 24, 10745 .

[46] Xu, Z.; Kim, S. K.; Yoon, J. Chem. Soc. Rev. 2010, 39, 1457.

[47] Xu, Z.; Singh, N. J.; Lim, J.; Pan, J.; Kim, H. N.; Park, S.; Kim, K. K.; Yoon, J. J. Am. Chem. Soc. 2009, 131, 15528.

[48] Farshbaf, S.; Anzenbacher, P. Jr. Chem. Commun. 2019, 55, 1770.

[49] Luo, J.; Xie, Z.; Lam, J. W. Y.; Chen, L.; Chen, H.; Qiu, C.; Kwok, H. S.; Zhan, X.; Liu, Y.; Zhu, D.; Tang, B. Z. Chem. Commun. 2001, 1740.

[50] Kwok, R. T.; Leung, C. W.; Lam, J. W.; Tang, B. Z. Chem. Soc. Rev 2015, 44, 4228.

[51] Xu, W.; Lee, M. M. S.; Zhang, Z.; Sung, H. H. Y.; Williams, I. D.; Kwok, R. T. K.; Lam, J. W. Y.; Wang, D.; Tang, B. Z. Chem. Sci. 2019, 10, 3494.

[52] Li, X.; Guo, X.; Cao, L.; Xun, Z.; Wang, S.; Li, S.; Li, Y.; Yang, G. Angew. Chem. Int. Ed. 2014, 53, 7809.

[53] Yang, Y.; Wang, X.; Cui, Q.; Cao, Q.; Li, L. ACS. Appl. Mater. Interfaces. 2016, 8, 7440.

[54] Cheng, L.; Zhang, H.; Dong, Y.; Zhao, Y.; Yu, Y.; Cao, L. Chem. Commun. 2019, 55, 2372.

[55] Jiang, G.; Zhu, W.; Chen, Q.; Shi, A.; Wu, Y.; Zhang, G.; Li, X.; Li, Y.; Fan, X.; Wang, J. Analyst 2017, 142, 4388.

[56] Noguchi, T.; Shiraki, T.; Dawn, A.; Tsuchiya, Y.; Lien, L. T. N.; Yamamoto, T.; Shinkai, S. Chem. Commun. 2012, 48, 8090.

[57] Maity, D.; Li, M.; Ehlers, M.; Schmuck, C. Chem. Commun. 2016, 53,208

[58] Tan, K. Y.; Li, C. Y.; Li, Y. F.; Fei, J.; Yang, B.; Fu, Y. J.; Li, F. Anal. Chem. 2017, 89, 1749

[59] Tang, J. L.; Li, C. Y.; Li, Y. F.; Zou, C. X. Chem. Commun. 2014, $50,15411$.

[60] Fang, Y.; Shi, W.; Hu, Y.; Li, X.; Ma, H. Chem. Commun. 2018, 54, 5454.

[61] Wang, L.; Yuan, L.; Zeng, X.; Peng, J.; Ni, Y.; Er, J. C.; Xu, W.; Agrawalla, B. K.; Su, D.; Kim, B.; Chang, Y. T. Angew. Chem. Int Ed. 2016, 55, 1773.

[62] Das, S.; Sarkar, H. S.; Uddin, M. R.; Rissanen, K.; Madal, S.; Sahoo, P. Chem. Commun. 2017, 53, 7600.

[63] Liu, Y.; Lee, D.; Wu, D.; Swamy, K. M. K.; Yoon, J. Sensors Actuators, $B$ 2018, 265, 429.

[64] Zhang, P.; Zhu, M.; Luo, H.; Zhang, Q.; Guo, L. E.; Li, Z.; Jiang, Y. B. Anal. Chem. 2017, 89,6210 . 